Draft Version September 1, 2020

Typeset using LATEX twocolumn style in AASTeX63

\title{
The First Mid-Infrared Detection of HNC in the Interstellar Medium: Probing the Extreme Environment Towards the Orion Hot Core
}

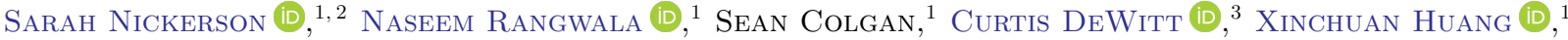 \\ Kinsuk Acharyya (D), ${ }^{4}$ Maria Drozdovskaya (D), ${ }^{5}$ Ryan C. Fortenberry (D),${ }^{6}$ Eric Herbst $\left(\mathbb{D},{ }^{7}\right.$ And \\ Timothy J. LeE (D) \\ ${ }^{1}$ Space Science and Astrobiology Division, NASA Ames Research Center, Moffet Field, CA, 94035 USA \\ ${ }^{2}$ Bay Area Environmental Research Institute, Moffet Field, CA, 94035, USA \\ ${ }^{3}$ USRA, SOFIA, NASA Ames Research Center MS 232-11, Moffett Field, CA 94035, USA \\ ${ }^{4}$ Physical Research Laboratory: Ahmedabad, Gujarat, India \\ ${ }^{5}$ Center for Space and Habitability, University of Bern, Gesellschaftsstrasse 6, CH-3012 Bern, Switzerland \\ ${ }^{6}$ Department of Chemistry and Biochemistry, University of Mississippi, 38655 USA \\ ${ }^{7}$ Departments of Chemistry and Astronomy, University of Virginia, McCormick Rd, Charlottesville, VA, 22904 USA
}

\begin{abstract}
We present the first mid-infrared (MIR) detections of $\mathrm{HNC}$ and $\mathrm{H}^{13} \mathrm{CN}$ in the interstellar medium, and numerous, resolved HCN rovibrational transitions. Our observations span 12.8 to $22.9 \mu \mathrm{m}$ towards the hot core Orion IRc2, obtained with the Echelon-Cross-Echelle Spectrograph aboard the Stratospheric Observatory for Infrared Astronomy (SOFIA). Exceptional, $\sim 5 \mathrm{~km} \mathrm{~s}^{-1}$ resolution distinguishes individual rovibrational transitions of the HNC and HCN P, Q, and R branches; and the $\mathrm{H}^{13} \mathrm{CN} \mathrm{R}$ branch. This allows direct measurement of the species' excitation temperatures, column densities, and relative abundances. $\mathrm{HNC}$ and $\mathrm{H}^{13} \mathrm{CN}$ exhibit a local standard rest velocity of $-7 \mathrm{~km} \mathrm{~s}^{-1}$ that may be associated with an outflow from nearby radio source I and an excitation temperature of about $100 \mathrm{~K}$. We resolve two velocity components for $\mathrm{HCN}$, the primary component also being at -7 $\mathrm{km} \mathrm{s}^{-1}$ with temperature $165 \mathrm{~K}$. The hottest component, which had never before been observed, is at $1 \mathrm{~km} \mathrm{~s}^{-1}$ with temperature $309 \mathrm{~K}$. This is the closest component to the hot core's centre measured to date. The derived ${ }^{12} \mathrm{C} /{ }^{13} \mathrm{C}=13 \pm 2$ is below expectation for Orion's Galactocentric distance, but the derived $\mathrm{HCN} / \mathrm{HNC}=72 \pm 7$ is expected for this extreme environment. Compared to previous sub-mm and mm observations, our SOFIA line survey of this region shows that the resolved MIR molecular transitions are probing a distinct physical component and isolating the chemistry closest to the hot core.
\end{abstract}

\section{INTRODUCTION}

The isomers hydrogen cyanide (HCN) and hydrogen isocyanide (HNC) have shown their ubiquity through a range of astrophysical phenomena since their first detection in Galactic star-forming regions (Snyder \& Buhl 1971; Zuckerman et al. 1972; Snyder \& Buhl 1972; McGuire 2018). Galactic sources include comets in our Solar System (Lis et al. 1997; Agúndez et al. 2014), Titan's atmosphere (Moreno et al. 2011), dark molecular clouds (Irvine \& Schloerb 1984; Hirota et al. 1998), diffuse clouds (Turner et al. 1997; Liszt \& Lucas 2001), star-forming regions (Loughnane et al. 2012; Tennekes

Corresponding author: Sarah Nickerson

sarah.nickerson@nasa.gov et al. 2006), protoplanetary objects (Dutrey et al. 1997; Kastner et al. 1997; Hrivnak et al. 2000; Herpin \& Cernicharo 2000; Graninger et al. 2015), circumstellar envelopes (Bujarrabal et al. 1994; Cernicharo et al. 1996, 2013), carbon stars (Harris et al. 2003) and the Galactic centre's circumnuclear disk (Harada et al. 2015). The isotopes of HCN and HNC are useful for measuring nitrogen, carbon, and hydrogen isotopic ratios in starforming regions and cores (Wampfler et al. 2014; Zeng et al. 2017; Colzi et al. 2018b,a). They also play a role in grain-surface chemistry at cold temperatures (Lo et al. 2015). HCN and HNC have also been detected in several external galaxies (Rickard et al. 1977; Henkel et al. 1988; Gao \& Solomon 2004) including Seyfert galaxies (PérezBeaupuits et al. 2007), molecular outflows (Aalto et al. 2012), and high redshift galaxies (Guélin et al. 2007). 
Several studies have also discovered HCN or HNC in hot cores (Goldsmith et al. 1981; Schilke et al. 1992; Lahuis \& van Dishoeck 2000; Knez et al. 2001; Boonman et al. 2001; Lacy et al. 2002; Knez et al. 2009; Rolffs et al. 2011). These warm ( $\geq 100 \mathrm{~K})$, dense $\left(10^{5}\right.$ to $10^{8} \mathrm{~cm}^{-3}$ ) regions of the interstellar medium (ISM) near young, high-mass protostars. Stellar radiation heats the gas and dust grains; evaporates the icy dust mantles in the cold molecular cloud where the protostar formed; and reveals a chemically rich reservoir of complex organic molecules (Ohishi 1997; van der Tak 2004; Bisschop et al. 2007; Belloche et al. 2013; Rivilla et al. 2017). Hot cores are possibly the antecedents to later ultra compact HiI regions (Cesaroni 2005), representing a key stage in stellar evolution. Similar hot corinos envelop low-mass protostars (Bottinelli et al. 2004).

The HCN/HNC abundance ratio changes under different conditions in star-forming regions, providing a useful probe of regional properties. HCN/HNC nears unity at low temperatures (Irvine \& Schloerb 1984; Schilke et al. 1992), infrared dark clouds (Vasyunina et al. 2011; Miettinen 2014) and dark cores (Hirota et al. 1998). Dark cores are defined as regions with temperatures $\sim 10 \mathrm{~K}$ and densities of $10^{3}$ to $10^{5} \mathrm{~cm}^{-3}$ (Benson \& Myers 1989). By comparison, in the Orion Molecular Cloud (OMC1) region, the $\mathrm{HCN}$ abundance remains similar to these dark cores while the HNC abundance drops. $\mathrm{HCN} / \mathrm{HNC}$ is $\sim 80$ towards the Orion hot core IRc2, and drops to $\sim 5$ away from the hot core (Schilke et al. 1992). Later observations in this region found a correlation between gas kinetic temperature and the HCN-to-HNC intensity ratio (Hacar et al. 2020).

These isomers are a promising chemical clock. Jin et al. (2015) measured the HCN/HNC abundance ratio in massive star-forming regions at differing evolutionary stages (infrared dark clouds, high-mass protostellar objects, and ultra-compact HiI regions), revealing that this ratio increases towards more advanced stages. Indeed, the evidence suggests that $\mathrm{HNC}$ is more prone to destruction at higher temperatures than $\mathrm{HCN}$, and will be less abundant in more advanced protostellar objects as they heat the ISM.

Theoretical calculations predict the isomers' main formation pathway to be the dissociative recombination of $\mathrm{HCNH}^{+}$with an electron (Herbst 1978), and experiments find this produces near equal quantities of $\mathrm{HCN}$ and HNC (Mendes et al. 2012). However, HNC is the the less stable of the two isomers (Lee \& Rendell 1991; Bowman et al. 1993; Nguyen et al. 2015) and should be even less abundant than observed (for an overview see Loison et al. 2014). Mechanisms proposed to regulate the $\mathrm{HCN} / \mathrm{HNC}$ ratio include the gas-phase $\mathrm{H}+$
HNC reaction barrier (Graninger et al. 2014), UV dissociation (Chenel et al. 2016; Aguado et al. 2017), and collisions with $\mathrm{H}_{2}$ (Hernández Vera et al. 2017) and He (Sarrasin et al. 2010).

Despite being the first hot core discovered (Ho et al. 1979), Orion IRc2 is atypical. Most hot cores are mainly internally heated, enveloping their protostar, while IRc2 is heated externally without evidence of any internal source (Blake et al. 1996; Okumura et al. 2011). A previous explosive outflow may have heated it while it was a preexisting dense clump, it may be itself an explosive outflow, or it is possibly a cavity offering a glimpse into a dust obscured protostar (Shuping et al. 2004; Zapata et al. 2011; Goddi et al. 2011; Bally et al. 2017; OrozcoAguilera et al. 2017). The main candidate for IRc2's heat source is radio source I, a possible binary system of protostars (Hirota et al. 2017), which is heavily dustobscured (Plambeck \& Wright 2016) and considered to be the main energy source of the region (Hirota et al. 2015). Nonetheless IRc2's location in the Orion Molecular Cloud, the nearest massive star-forming region to Earth at $388 \pm 5$ pc (Kounkel et al. 2017), makes it ideal for observing the rich hot core chemistry.

Numerous HCN and HNC emission lines have been observed towards IRc2 in the mm (Goldsmith et al. 1986; Schilke et al. 1992) and sub-mm (Stutzki et al. 1988; Harris et al. 1995; Schilke et al. 2001; Comito et al. 2005), two well-studied spectral regions due to to their accessibility from the ground. However, observations in the mid-infrared (MIR) have been scarce due to atmospheric absorption. The MIR is nonetheless critical for understanding fully the chemistry of the ISM. Additionally, rovibrational transitions for molecules with no permanent dipole moment are accessible only in the this wavelength range.

The Short-Wavelength Spectrometer aboard the Infrared Space Observatory covered 2.38 to $45.2 \mu \mathrm{m}$ (De Graauw et al. 1996), but detection was limited to the strongest absorption features and not individual rovibrational transitions in hot cores (van Dishoeck et al. 1998; Lahuis \& van Dishoeck 2000; Boonman et al. 2003). Similarly, Spitzer's Infrared Spectrograph (Houck et al. 2004) could only detect the strongest HCN absorption feature towards young stellar objects (An et al. 2009; Lahuis et al. 2006; An et al. 2011). The ground-based Texas Echelon Cross Echelle Spectrograph (TEXES, Lacy et al. 2002) resolves these individual transitions from 5 to $25 \mu \mathrm{m}$ with a maximum resolving power of $\sim 100,000$, but TEXES cannot access the entire MIR range due to atmospheric interference. HCN rovibrational and rotational transitions were detected with TEXES towards the hot cores AFGL 2591 (Knez et al. 
2001), NGC 7538 IRS 1 (Knez et al. 2009), and IRc2 (Lacy et al. 2002; Lacy et al. 2005).

The Stratospheric Observatory for Infrared Astronomy (SOFIA, Young et al. 2012) is an airbourne observatory that flies above $99 \%$ of atmospheric water vapour. The Echelon-Cross Echelle Spectrograph instrument (EXES, Richter et al. 2018) aboard SOFIA observes in MIR from 5 to $28 \mu \mathrm{m}$ with a spectral resolution of $10^{3}$ to $10^{5}$. Currently, this is the only spectrograph able to resolve individual molecular transitions over the entire MIR. Several studies of hot cores with SOFIA/EXES are ongoing (Indriolo et al. 2015a; Barr et al. 2018; Dungee et al. 2018; Indriolo et al. 2020; Barr et al. 2020). Rangwala et al. (2018) detected eight HCN $\mathrm{R}$ band rovibrational transitions towards IRc2 between 12.96 and $13.33 \mu \mathrm{m}$, enough to directly calculate its temperature and column density.

In this work, we present high-resolution observations of $\mathrm{HCN}, \mathrm{H}^{13} \mathrm{CN}$, and $\mathrm{HNC}$ towards the hot core Orion IRc2 from 12.8 to $22.9 \mu \mathrm{m}$. This is the first ISM detection of $\mathrm{HNC}$ and $\mathrm{H}^{13} \mathrm{CN}$ in the MIR. Additionally, at MIR wavelengths, SOFIAs beam size $\left(33^{\prime \prime} 2 \times 3^{\prime \prime} \cdot 2\right)$ is much smaller compared with space-based missions like ISO $\left(14^{\prime \prime} \times 27^{\prime \prime}\right)$ and most previous sub- $\mathrm{mm} / \mathrm{mm}$ studies from the ground. This enables us to unambiguously isolate a previously unprobed, hotter component of molecular gas, traced by HCN, closest to the hot core from the surrounding emission.

We provide our observational methods in $\S 2$, detail our analysis in $\S 3$, discuss the implications of the results and compare them with both other observations and models in $\S 4$, and summarize our conclusions in $\S 5$. Our observations are part of a wider molecular survey of Orion IRc2 in the MIR from 7.2 to 8 and 13.2 to 28.3 $\mu \mathrm{m}$ (publication in preparation).

\section{OBSERVATIONS AND DATA REDUCTION}

We observed Orion IRc2 with the EXES instrument aboard the SOFIA observatory between 2018 October 27 and 31 at altitudes from about 42,000 to $44,000 \mathrm{ft}$ in High-low mode. Spectra were acquired in the crossdispersed high-resolution mode with a slit width of 3.2 giving a resolving power of about $60,000\left(\sim 5 \mathrm{~km} \mathrm{~s}^{-1}\right)$. We used the cross-disperser grating in 1st order to obtain the broadest simultaneous wavelength coverage per spectral setting. The length of the slit varied between $1^{\prime \prime} 9$ and $6 . " 9$, depending on the spectral setting. Table 1 gives the details for these six settings, each of which is split into several orders. For all observations, we nodded the telescope to an off-source position relatively free of emission $15^{\prime \prime}$ East and 25".9 North of IRc2, at 1 minute intervals, in order to remove sky emission and thermal background from the telescope system.

We reduce the EXES data with the SOFIA Redux pipeline (Clarke et al. 2015). Wavelength scales are calibrated using sky emission line spectra produced for each setting by omitting the nod subtraction step and then adjusting the scale to match observed sky emission line wavelengths to their values in the HITRAN database (Gordon et al. 2017). The absolute velocity uncertainty is 0.15 to $0.3 \mathrm{~km} \mathrm{~s}^{-1}$, estimated from High-Medium mode sky lines of other settings. Figure 1 gives the EXES footprint for each observational setting over SOFIA/FORECAST maps at 7.7 and $19.7 \mu \mathrm{m}$ (De Buizer et al. 2012). Each observation is centred over IRc2, and we also show the positions of IR sources IRc4, IRc7, and BN, and radio source I, which has no IR component and may be heating IRc2. For settings 445 and $460 \mathrm{~cm}^{-1}$, in particular, we were concerned about contamination and dilution from IRc7. However, after examining spectra split along the slit width, we confirm that the spectral lines originate from the slit centre, over IRc2, and not the edge over IRc7. Furthermore, from ground-based MIR spectroscopy of IRc2 and IRc7, Evans et al. (1991) found that $\mathrm{C}_{2} \mathrm{H}_{2}$ and $\mathrm{HCN}$ have 2 and 3 times higher column densities, respectively, towards IRc2 than IRc7. Therefore, our observations are centred over IRc2 and are unlikely to be contaminated by IRc7.

We find HCN in two settings covering 701 to $783 \mathrm{~cm}^{-1}$ (12.8 to $14.3 \mu \mathrm{m}), \mathrm{H}^{13} \mathrm{CN}$ in one setting from 701 to 725 $\mathrm{cm}^{-1}$ (13.5 to $14.3 \mu \mathrm{m}$ ), and HNC in four settings from 436 to $498 \mathrm{~cm}^{-1}$ (20.1 to $\left.22.9 \mu \mathrm{m}\right)$. We searched for, but do not observe, the isotopologues $\mathrm{HC}^{15} \mathrm{~N}$ and $\mathrm{DCN}$ with the HITRAN (Gordon et al. 2017) and GEISA (Jacquinet-Husson et al. 2016) databases respectively and $\mathrm{HN}^{13} \mathrm{C}$ and $\mathrm{H}^{15} \mathrm{NC}$ with our own theoretically calculated line lists. Figure 2 shows an example of spectra for the 724 and $460 \mathrm{~cm}^{-1}$ settings with lines of $\mathrm{HCN}$, $\mathrm{H}^{13} \mathrm{CN}$, and HNC.

\section{ANALYSIS \\ 3.1. Peak Finding}

In order to normalize our fluxes we must first identify the peaks and the baseline of each order. We employ a simple peak-finding algorithm to identify minima and maxima. For a given order, we perform Gaussian smoothing on the flux and then take the sign of the derivative of the smoothed flux. We consider blocks of consecutive pixels with the same sign. If two blocks of opposite signs are adjacent, or have a small gap between them, they are considered to belong to a peak and the peak position is taken to be the centre of the gap be- 

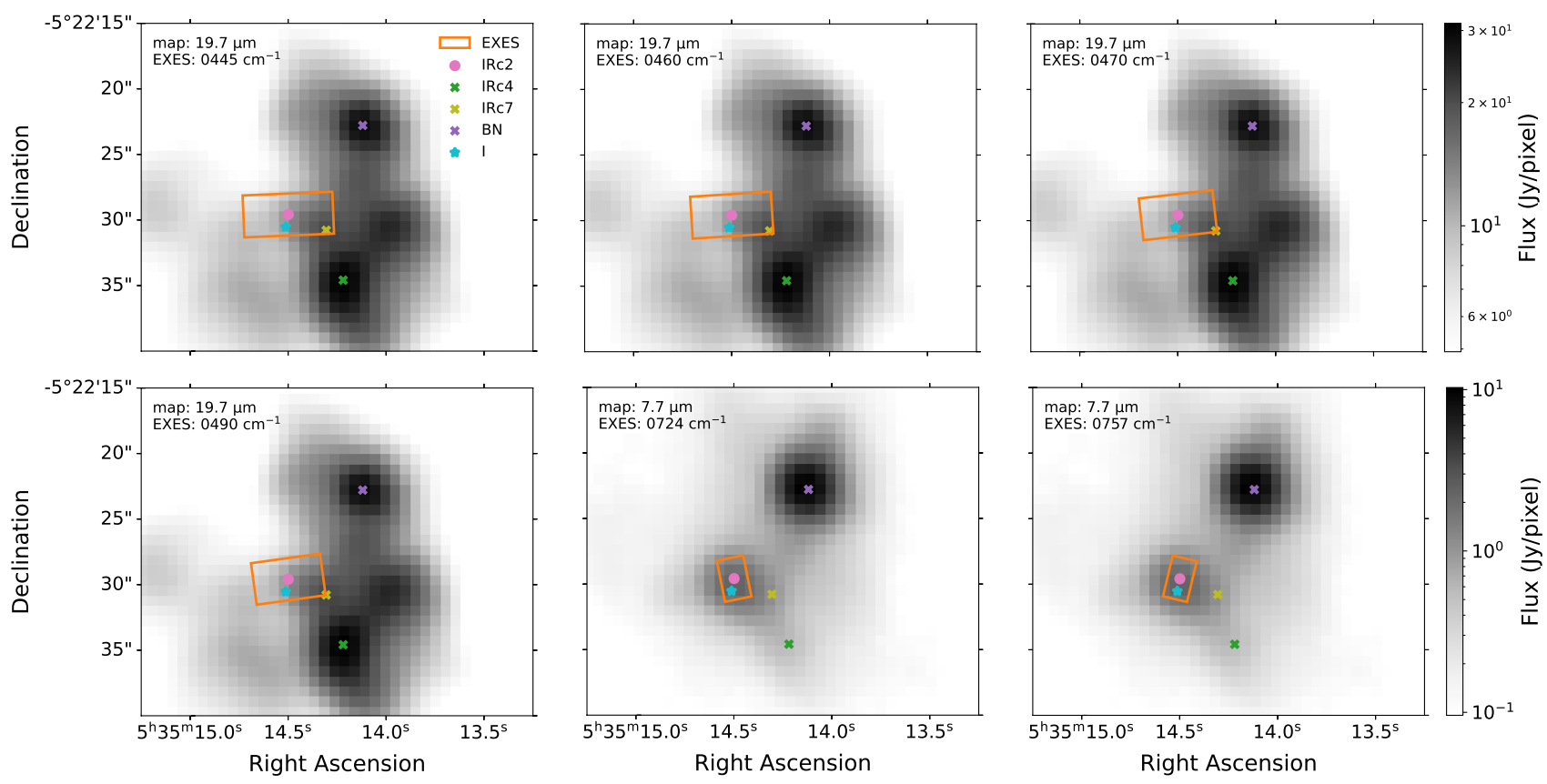

Figure 1. The EXES footprint for each setting superimposed on an SOFIA/FORECAST map of the region (De Buizer et al. 2012). In addition to our target, IRc2, the positions of radio source I and nearby IR sources IRc4, IRc7, and BN are given. Note in order to display maps closest in wavelength to each EXES setting, that $7.7 \mu \mathrm{m}$ map is for the 700 settings, and the 19.7 $\mu \mathrm{m}$ map is for the 400's $\mathrm{cm}^{-1}$ setting. Their flux scales differ, given by the greyscale bars on the right.

Table 1. Specifications for each EXES setting

\begin{tabular}{cccccccc}
\hline \hline $\begin{array}{c}\text { Setting } \\
\left(\mathrm{cm}^{-1}\right)\end{array}$ & Species & $\begin{array}{c}\text { Min } \lambda \\
(\mu \mathrm{m})\end{array}$ & $\begin{array}{c}\text { Max } \lambda \\
(\mu \mathrm{m})\end{array}$ & $\begin{array}{c}\text { Date } \\
(\text { yyyy-mm-dd })\end{array}$ & $\begin{array}{c}\text { Altitude } \\
(\mathrm{ft})\end{array}$ & $\begin{array}{c}\text { Slit Length } \times \text { Width } \\
\left({ }^{\prime \prime} \times{ }^{\prime \prime}\right)\end{array}$ & $\begin{array}{c}\text { Integration Time } \\
(\mathrm{s})\end{array}$ \\
\hline 757 & $\mathrm{HCN}$ & 12.8 & 13.6 & $2018-10-31$ & 42,008 & $1.9 \times 3.2$ & 2,560 \\
724 & $\mathrm{HCN}, \mathrm{H}^{13} \mathrm{CN}$ & 13.5 & 14.3 & $2018-10-30$ & 44,002 & $2.1 \times 3.2$ & 2,880 \\
490 & $\mathrm{HNC}$ & 20.1 & 20.8 & $2018-10-27$ & 43,014 & $5.3 \times 3.2$ & 576 \\
470 & $\mathrm{HNC}$ & 20.8 & 21.5 & $2018-10-27$ & 43,015 & $5.7 \times 3.2$ & 512 \\
460 & $\mathrm{HNC}$ & 21.5 & 22.2 & $2018-10-27$ & 42,010 & $6.2 \times 3.2$ & 512 \\
445 & $\mathrm{HNC}$ & 22.2 & 22.9 & $2018-10-27$ & 42,005 & $6.9 \times 3.2$ & 512 \\
\hline
\end{tabular}

tween them. If a block is next to an order endpoint, then that endpoint is considered a peak. Three input parameters to the peak-finder, on occasion, require adjustment depending on the order: sigma for strength of Gaussian smoothing, number of consecutive pixels with the same sign to be considered a block, and the size of the gap between blocks of consecutive pixels.

\subsection{Normalization and Atmospheric Line Correction}

While the lines in settings 445, 460, 470, and $490 \mathrm{~cm}^{-1}$ are clear of atmospheric features, many lines in 724 and $757 \mathrm{~cm}^{-1}$ are mixed with atmospheric lines, necessitating correction (for example: HCN Q8e, top panel Figure
2). We download the unsmoothed ATRAN atmospheric model (Lord 1992) ${ }^{1}$ for each setting given their altitude, latitude, and zenith of observation. This unsmoothed ATRAN model corresponds to infinite resolution. To divide the ATRAN from our flux, we seek to smooth ATRAN to EXES's resolution and normalize our flux to ATRAN simultaneously.

Each order requires a different normalization, especially the settings 445 to $490 \mathrm{~cm}^{-1}$ while an entire setting should have the same resolution. To achieve this,

\footnotetext{
${ }^{1}$ https://atran.arc.nasa.gov/cgi-bin/atran/atran.cgi
} 

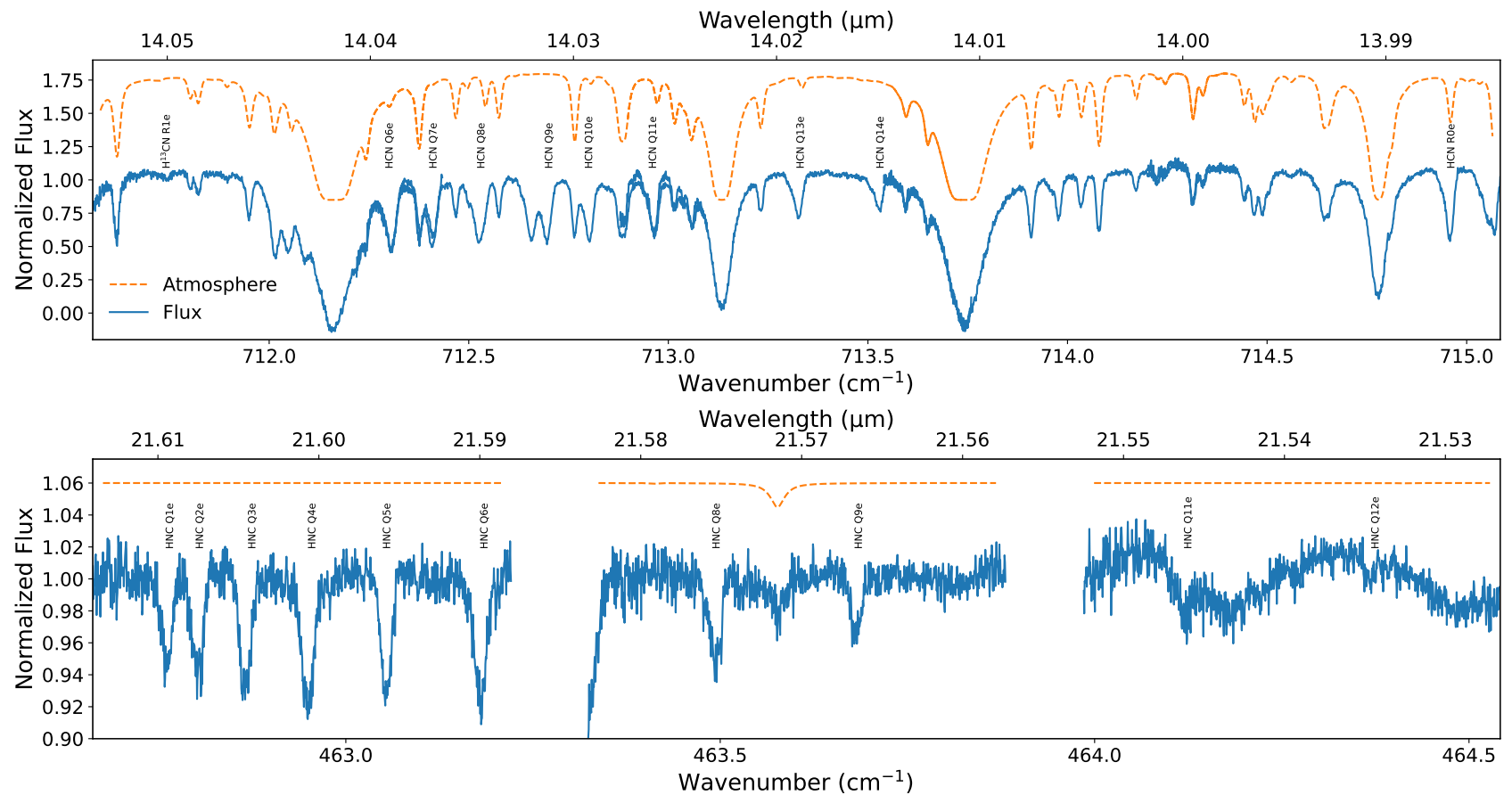

Figure 2. Sample of EXES spectra for HCN (top), $\mathrm{H}^{13} \mathrm{CN}$ (top), and HNC (bottom). The flux is normalized and the atmosphere smoothed according to $\S 3.2$ and offset for display purposes. Note that in the top panel, lines overlap from adjacent orders. The bottom panel shows three separated orders, the leftmost two of which have been baseline corrected by a polynomial, while the rightmost has not due to the absence of a satisfactory polynomial.

we implement the following procedure on the fluxes from each order:

1. For each individual order, we run the peak finder described in $\S 3.1$ on the observed EXES flux and the ATRAN model.

2. Due to standing waves in settings 445 and 460 $\mathrm{cm}^{-1}$, a few orders require baseline correction where possible (see Figure 2, bottom panel). Considering all pixels not in a peak to be part of the baseline, we fit the baselines of these orders to a polynomial, $n_{b}$, and divide the flux by this polynomial in order to straighten the baseline.

3. We mask peaks that appear in the EXES flux and not in the ATRAN model, as well as deep $\mathrm{CO}_{2}$ lines that bottomed out at 0 in ATRAN but extended to negative in EXES (see Figure 2, top panel, for three examples). This leaves us with fluxes $F_{A}$ from ATRAN and $F_{E}$ from EXES that match.

4. We conduct the following procedure on each order:

$$
\min \left[\sum_{i}^{\text {pixels }}\left\|\operatorname{G1d}\left[F_{A, i}, \sigma_{G}\right]-\frac{F_{E, i}}{n_{b, i} n_{\mathrm{c}}}\right\|\right],
$$

where $F_{A, i}$ is the ATRAN model flux at pixel $i, F_{E, i}$ is the our observed EXES flux at pixel $i, n_{b, i}$ is the normalization polynomial's value at pixel $i$ if needed (otherwise $n_{b, i}=1$, see step 2 above), $n_{c}$ is the normalization constant, min is the routine optimize.minimize from the scipy python package (Virtanen et al. 2020) that minimizes its enclosed function, and G1d is the routine scipy.ndimage.gaussian_filter $1 d$ that smooths its enclosed function with standard deviation $\sigma_{G} . F_{A, i}, F_{E, i}$, and $n_{b, i}$ are inputs to Equation 1 , and min returns the values of $n_{c}$ and $\sigma_{G}$ that minimize Equation 1. We are finding the resolution and flux normalization that minimizes the difference between the ATRAN model and our observed EXES flux.

5. We retain $n_{c}$ for each individual order, but discard $\sigma_{G}$, because many orders do not have enough, or any, ATRAN lines to match accurately the resolution. With each order normalized individually, we input the fluxes from an entire setting back into Equation 1 to obtain that setting's overall $\sigma_{G}$.

6. We divide the order-normalized EXES flux by the setting-wide smoothed ATRAN model to correct for the atmosphere and use this flux for line fitting in $\S 3.3$. 


\subsection{Line Fitting}

We rerun the peak finder from $\S 3.1$ again on the normalized flux to match these peaks with the molecular absorption lines of interest. In many cases, this is sufficient to correctly identify the lines' extent. We only need to manually identify line boundaries in cases where the line was either weak or one of many in a crowded region. For settings 724 and $757 \mathrm{~cm}^{-1}$, we also correct for atmosphere as in $§ 3.2$, while this is unnecessary for the other settings as no lines are near to any atmospheric absorption.

With scipy.optimize.curvefit, we fit the measured absorption lines with a Gaussian profile following Indriolo et al. (2015b):

$$
I=I_{0} e^{-\tau_{0} G},
$$

where,

$$
G=\exp \left[-\frac{\left(v-v_{c}\right)^{2}}{2 \sigma_{v}^{2}}\right]
$$

$I_{0}$ is the normalized continuum level (typically close to unity), $\tau_{0}$ is the line centre optical depth, $v$ is the local standard of rest (LSR) velocity, $v_{c}$ is the velocity of the line centre, and $\sigma_{v}$ is the velocity dispersion. In the cases of double Gaussians, 1 and 2, we fit:

$$
I=I_{0} e^{-\left(\tau_{01} G_{1}+\tau_{02} G_{2}\right)} .
$$

Triple Gaussians follow a similar equation. Each HNC and $\mathrm{H}^{13} \mathrm{CN}$ line is best fit by a single Gaussian. Almost all HCN lines show two velocity components and are best fit by a double Gaussian with a handful of exceptions. P3e, R17e, and R18e are not resolved enough for a double Gaussian. Q9e and R12e overlap with acetylene absorption lines, leaving single Gaussians. Q8e, Q11e, Q14e and R6e overlap with atmospheric lines that are divided out. However, due to the ATRAN model not matching the data exactly, the remaining flux is slightly distorted and a triple Gaussian fits these lines best to correct for this distortion.

Figure 3 shows six examples of fits, with single Gaussians for $\mathrm{HNC}$ and $\mathrm{H}^{13} \mathrm{CN}$, and double or triple Gaussians for HCN. HCN R10e is an example of where the secondary velocity component is distinct. While $\mathrm{HCN}$
Q7e is similarly best fit by a double Gaussian, an atmospheric line falls over the secondary component and little remains after atmospheric correction. In cases such as this, we consider the entire line as belonging to the primary velocity component, even though some trace of the secondary remains. Q8e is an example of a triple Gaussian, where even after atmospheric correction the primary Gaussian is distorted and two Gaussians fit it best. Another Gaussian fits the secondary velocity component.

We calculate the column density, $N_{l}$, in the lower state of an observed transition from the integral over the absorption line:

$$
\begin{aligned}
d N_{l} / d v & =\frac{g_{l}}{g_{u}} \frac{8 \pi}{A \lambda^{3}} \tau_{0} G, \\
N_{l} & =\sqrt{2 \pi} \frac{g_{l}}{g_{u}} \frac{8 \pi}{A \lambda^{3}} \tau_{0} \sigma_{v},
\end{aligned}
$$

where $g_{l}$ and $g_{u}$ are the lower and upper statistical weights respectively, $A$ is the Einstein constant for spontaneous emission, and $\lambda$ is the rest wavelength of the transition. We obtain these parameter values (along with $E_{l}$ required in §3.4) for $\mathrm{HCN}$ and $\mathrm{H}^{13} \mathrm{CN}$ from the HITRAN database (Gordon et al. 2017) and for HNC from the GEISA database (Jacquinet-Husson et al. 2016).

Tables 2, 3, and 4 list the best-fit parameters with fitted errors as well as the relevant molecular database parameters for $\mathrm{HNC}, \mathrm{H}^{13} \mathrm{CN}$, and $\mathrm{HCN}$ respectively. All three species transition from the ground state to the $\nu_{2}$ band. A number of lines are missing due to gaps between orders or heavy interference from atmospheric and acetylene lines.

In the case of $\mathrm{HCN}$, a superscript on the column density indicates to which velocity component the Gaussian fit belongs. These components separately contribute to rotation diagrams in $\S 3.4$. For components with more than one Guassian fit, the column density is the total of the Gaussian fits comprising it, while the velocity will be the velocity of the component with the highest optical depth. This is because the shallower components are artifacts of atmospheric lines. 
Table 2. Observed $\nu_{2}$ band HNC Transitions and Inferred Parameters

\begin{tabular}{|c|c|c|c|c|c|c|c|c|}
\hline Transition & $\begin{array}{l}\text { Wavenumber } \\
\qquad\left(\mathrm{cm}^{-1}\right)\end{array}$ & $\begin{array}{c}E_{l} / k_{b} \\
(\mathrm{~K})\end{array}$ & $g_{l}$ & $\begin{array}{c}A \\
\left(\mathrm{~s}^{-1}\right)\end{array}$ & $\begin{array}{c}v_{\mathrm{LSR}} \\
\left(\mathrm{km} \mathrm{s}^{-1}\right)\end{array}$ & $\begin{array}{c}v_{\mathrm{FWHM}} \\
\left(\mathrm{km} \mathrm{s}^{-1}\right)\end{array}$ & $\tau_{0}$ & $\begin{array}{c}N_{l} \\
\times 10^{14} \mathrm{~cm}^{-2}\end{array}$ \\
\hline P3e & 453.64798 & 26.1 & 42 & 1.269 & $-6.8 \pm 0.3$ & $12.6 \pm 1.5$ & $0.031 \pm 0.003$ & $1.09 \pm 0.20$ \\
\hline $\mathrm{P} 5 \mathrm{e}$ & 447.5964 & 65.3 & 66 & 1.352 & $-7.0 \pm 0.2$ & $10.6 \pm 0.5$ & $0.041 \pm 0.001$ & $0.94 \pm 0.05$ \\
\hline $\mathrm{P} 6 \mathrm{e}$ & 444.57022 & 91.4 & 78 & 1.353 & $-5.3 \pm 0.4$ & $14.4 \pm 2.0$ & $0.029 \pm 0.003$ & $0.86 \pm 0.19$ \\
\hline $\mathrm{P} 7 \mathrm{e}$ & 441.54388 & 121.8 & 90 & 1.345 & $-9.1 \pm 0.3$ & $10.5 \pm 1.3$ & $0.031 \pm 0.003$ & $0.65 \pm 0.11$ \\
\hline $\mathrm{P} 8 \mathrm{e}$ & 438.51747 & 156.6 & 102 & 1.33 & $-7.9 \pm 0.3$ & $11.8 \pm 1.1$ & $0.028 \pm 0.002$ & $0.64 \pm 0.08$ \\
\hline Q1e & 462.74319 & 4.4 & 18 & 3.379 & $-8.9 \pm 0.2$ & $10.3 \pm 0.7$ & $0.057 \pm 0.003$ & $0.46 \pm 0.04$ \\
\hline Q2e & 462.78519 & 13.1 & 30 & 3.379 & $-8.1 \pm 0.2$ & $12.8 \pm 1.2$ & $0.071 \pm 0.004$ & $0.71 \pm 0.10$ \\
\hline Q3e & 462.84818 & 26.1 & 42 & 3.38 & $-8.9 \pm 0.1$ & $11.7 \pm 0.6$ & $0.080 \pm 0.003$ & $0.73 \pm 0.06$ \\
\hline $\mathrm{Q} 4 \mathrm{e}$ & 462.93214 & 43.5 & 54 & 3.382 & $-8.4 \pm 0.2$ & $11.6 \pm 0.6$ & $0.080 \pm 0.003$ & $0.72 \pm 0.05$ \\
\hline Q5e & 463.03705 & 65.3 & 66 & 3.383 & $-8.3 \pm 0.1$ & $10.7 \pm 0.4$ & $0.082 \pm 0.002$ & $0.69 \pm 0.04$ \\
\hline Q6e & 463.16287 & 91.4 & 78 & 3.385 & $-8.4 \pm 0.2$ & $13.2 \pm 0.5$ & $0.078 \pm 0.003$ & $0.81 \pm 0.04$ \\
\hline Q8e & 463.47714 & 156.6 & 102 & 3.39 & $-7.7 \pm 0.2$ & $12.6 \pm 1.0$ & $0.055 \pm 0.003$ & $0.54 \pm 0.06$ \\
\hline Q9e & 463.66551 & 195.8 & 114 & 3.393 & $-7.7 \pm 0.3$ & $10.5 \pm 1.0$ & $0.037 \pm 0.002$ & $0.30 \pm 0.04$ \\
\hline Q11e & 464.10446 & 287.1 & 138 & 3.4 & $-8.1 \pm 0.5$ & $7.7 \pm 2.5$ & $0.022 \pm 0.005$ & $0.13 \pm 0.07$ \\
\hline Q12e & 464.35494 & 339.3 & 150 & 3.404 & $-5.9 \pm 0.6$ & $8.6 \pm 1.6$ & $0.013 \pm 0.002$ & $0.09 \pm 0.02$ \\
\hline $\mathrm{R} 0 \mathrm{e}$ & 465.74576 & 0.0 & 6 & 2.298 & $-8.3 \pm 0.3$ & $11.9 \pm 1.3$ & $0.037 \pm 0.003$ & $0.17 \pm 0.03$ \\
\hline $\mathrm{R} 1 \mathrm{e}$ & 468.76863 & 4.4 & 18 & 2.113 & $-8.0 \pm 0.2$ & $14.6 \pm 0.7$ & $0.069 \pm 0.003$ & $0.79 \pm 0.05$ \\
\hline $\mathrm{R} 2 \mathrm{e}$ & 471.7907 & 13.1 & 30 & 2.053 & $-8.1 \pm 0.2$ & $12.3 \pm 0.8$ & $0.076 \pm 0.003$ & $0.92 \pm 0.08$ \\
\hline R3e & 474.8119 & 26.1 & 42 & 2.037 & $-8.0 \pm 0.2$ & $11.3 \pm 0.6$ & $0.081 \pm 0.003$ & $1.01 \pm 0.08$ \\
\hline $\mathrm{R} 5 \mathrm{e}$ & 480.85136 & 65.3 & 66 & 2.055 & $-8.2 \pm 0.2$ & $9.3 \pm 0.5$ & $0.071 \pm 0.003$ & $0.81 \pm 0.04$ \\
\hline $\mathrm{R} 7 \mathrm{e}$ & 486.88636 & 121.8 & 90 & 2.102 & $-7.7 \pm 0.3$ & $12.4 \pm 1.0$ & $0.048 \pm 0.003$ & $0.78 \pm 0.07$ \\
\hline $\mathrm{R} 8 \mathrm{e}$ & 489.902 & 156.6 & 102 & 2.131 & $-6.2 \pm 0.3$ & $9.2 \pm 1.0$ & $0.042 \pm 0.003$ & $0.51 \pm 0.08$ \\
\hline R9e & 492.91629 & 195.8 & 114 & 2.163 & $-7.8 \pm 0.3$ & $12.9 \pm 0.9$ & $0.043 \pm 0.002$ & $0.75 \pm 0.06$ \\
\hline $\mathrm{R} 10 \mathrm{e}$ & 495.92916 & 239.3 & 126 & 2.197 & $-5.9 \pm 0.5$ & $8.4 \pm 1.3$ & $0.026 \pm 0.004$ & $0.30 \pm 0.07$ \\
\hline
\end{tabular}

Note-Wavenumber is the rest wavenumber of the transition, $E_{l}$ is the energy level of the lower state, $k_{b}$ is the Boltzmann constant, $g_{l}$ is the lower statistical weight, $A$ is the Einstein constant, $v_{\mathrm{LSR}}$ is the observed local standard of rest velocity, $v_{\text {FWHM }}$ is the observed full-width half-maximum, $\tau_{0}$ is the observed optical depth, and $N_{l}$ is the observed column density of the transition. Data in the first five columns are from the GEISA database (Jacquinet-Husson et al. 2016).

Table 3. Observed $\nu_{2}$ band $\mathrm{H}^{13} \mathrm{CN}$ Transitions and Inferred Parameters

\begin{tabular}{|c|c|c|c|c|c|c|c|c|}
\hline Transition & $\begin{array}{l}\text { Wavenumber } \\
\qquad\left(\mathrm{cm}^{-1}\right)\end{array}$ & $\begin{array}{c}E_{l} / k_{b} \\
(\mathrm{~K})\end{array}$ & $g_{l}$ & $\begin{array}{c}A \\
\left(\mathrm{~s}^{-1}\right)\end{array}$ & $\begin{array}{c}v_{\mathrm{LSR}} \\
\left(\mathrm{km} \mathrm{s}^{-1}\right)\end{array}$ & $\begin{array}{c}v_{\mathrm{FWHM}} \\
\left(\mathrm{km} \mathrm{s}^{-1}\right)\end{array}$ & $\tau_{0}$ & $\begin{array}{c}N_{l} \\
\times 10^{14} \mathrm{~cm}^{-2}\end{array}$ \\
\hline R1e & 711.72312 & 4.1 & 36 & 1.206 & $-7.5 \pm 0.4$ & $8.7 \pm 0.9$ & $0.059 \pm 0.005$ & $2.46 \pm 0.27$ \\
\hline $\mathrm{R} 6 \mathrm{e}$ & 726.098321 & 87.0 & 156 & 1.143 & $-6.5 \pm 0.2$ & $8.2 \pm 0.6$ & $0.083 \pm 0.005$ & $5.30 \pm 0.44$ \\
\hline $\mathrm{R} 8 \mathrm{e}$ & 731.839651 & 149.2 & 204 & 1.157 & $-5.7 \pm 0.3$ & $7.0 \pm 0.8$ & $0.054 \pm 0.005$ & $3.05 \pm 0.40$ \\
\hline
\end{tabular}

Note-See Table 2 for column descriptions. Data in the first five columns are from the HITRAN database (Gordon et al. 2017). 

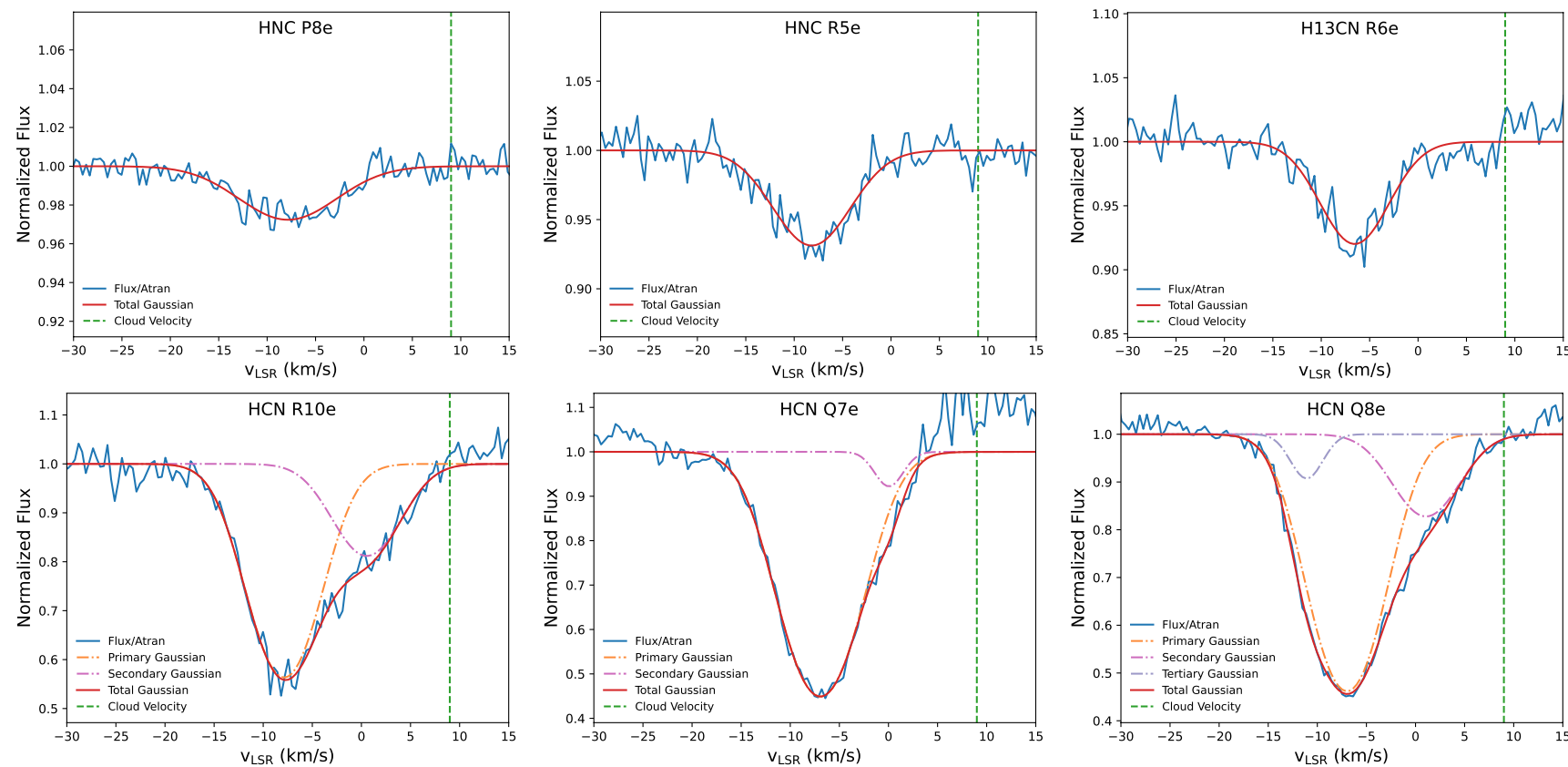

Figure 3. Gaussian fits for selected $\mathrm{HNC}$ (top left two), $\mathrm{H}^{13} \mathrm{CN}$ (top right), and $\mathrm{HCN}$ (bottom row) lines normalized to their baselines. The $\mathrm{HCN}$ and $\mathrm{H}^{13} \mathrm{CN}$ fluxes have been corrected for atmospheric absorption. $\mathrm{HNC}$ and $\mathrm{H}^{13} \mathrm{CN}$ are best fit by a single Gaussian, while HCN is best fit by a double Gaussian showing two velocity components. For the case of Q8e, two Gaussians are needed for the main velocity component to catch distortion from atmospheric division. The main velocity component of $\mathrm{HCN}$ is similar to that of $\mathrm{HNC}$ and $\mathrm{H}^{13} \mathrm{CN}$. The vertical dotted line indicates the systematic, ambient cloud velocity $9 \mathrm{~km} \mathrm{~s}$ (Zapata et al. 2012)

Table 4. Observed $\nu_{2}$ band HCN Transitions and Inferred Parameters

\begin{tabular}{|c|c|c|c|c|c|c|c|c|}
\hline Transition & $\begin{array}{c}\text { Wavenumber } \\
\qquad\left(\mathrm{cm}^{-1}\right)\end{array}$ & $\begin{array}{c}E_{l} / k_{b} \\
(\mathrm{~K})\end{array}$ & $g_{l}$ & $\begin{array}{c}A \\
\left(\mathrm{~s}^{-1}\right)\end{array}$ & $\begin{array}{c}v_{\mathrm{LSR}} \\
\left(\mathrm{km} \mathrm{s}^{-1}\right)\end{array}$ & $\begin{array}{c}v_{\mathrm{FWHM}} \\
\left(\mathrm{km} \mathrm{s}^{-1}\right)\end{array}$ & $\tau_{0}$ & $\begin{array}{c}N_{l} \\
\times 10^{14} \mathrm{~cm}^{-2}\end{array}$ \\
\hline \multirow[t]{2}{*}{$\mathrm{P} 2 \mathrm{e}$} & 706.0664 & 12.8 & 30 & 0.6576 & $-7.5 \pm 0.2$ & $7.8 \pm 0.3$ & $0.352 \pm 0.008$ & $65.46 \pm 3.32^{1}$ \\
\hline & & & & & $0.7 \pm 0.3$ & $7.5 \pm 0.6$ & $0.173 \pm 0.009$ & $30.99 \pm 3.46^{2}$ \\
\hline P3e & 703.109429 & 25.5 & 42 & 0.778 & $-6.8 \pm 0.3$ & $11.1 \pm 1.0$ & $0.429 \pm 0.029$ & $79.92 \pm 8.57^{1}$ \\
\hline \multirow[t]{2}{*}{ Q6e } & 712.286 & 89.3 & 78 & 2.028 & $-7.0 \pm 0.1$ & $9.7 \pm 0.3$ & $0.750 \pm 0.017$ & $34.76 \pm 1.11^{1}$ \\
\hline & & & & & - & $4.4 \pm 0.6$ & $0.180 \pm 0.019$ & $3.75 \pm 0.63^{1}$ \\
\hline \multirow[t]{2}{*}{ Q7e } & 712.388056 & 119.1 & 90 & 2.028 & $-6.9 \pm 0.1$ & $8.9 \pm 0.2$ & $0.801 \pm 0.011$ & $34.08 \pm 0.71^{1}$ \\
\hline & & & & & - & $3.4 \pm 0.8$ & $0.081 \pm 0.017$ & $1.29 \pm 0.43^{1}$ \\
\hline \multirow[t]{3}{*}{ Q8e } & 712.504639 & 153.1 & 102 & 2.028 & $-7.0 \pm 0.3$ & $8.3 \pm 0.3$ & $0.772 \pm 0.018$ & $30.55 \pm 1.60^{1}$ \\
\hline & & & & & $1.1 \pm 0.5$ & $7.7 \pm 0.8$ & $0.189 \pm 0.017$ & $6.97 \pm 1.22^{2}$ \\
\hline & & & & & - & $3.8 \pm 1.1$ & $0.09^{r} \pm 0.041$ & $1.76 \pm 1.18^{1}$ \\
\hline Q9e & 712.635726 & 191.4 & 114 & 2.028 & $-7.4 \pm 0.1$ & $12.4 \pm 0.2$ & $0.692 \pm 0.010$ & $41.06 \pm 0.68^{1}$ \\
\hline \multirow[t]{2}{*}{ Q10e } & 712.781294 & 233.9 & 126 & 2.027 & $-7.0 \pm 0.1$ & $8.5 \pm 0.2$ & $0.673 \pm 0.007$ & $27.23 \pm 0.53^{1}$ \\
\hline & & & & & - & $4.6 \pm 0.6$ & $0.117 \pm 0.009$ & $2.58 \pm 0.37^{1}$ \\
\hline \multirow[t]{3}{*}{ Q11e } & 712.941315 & 280.7 & 138 & 2.027 & $-7.4 \pm 0.1$ & $8.1 \pm 0.3$ & $0.592 \pm 0.011$ & $23.04 \pm 0.75^{1}$ \\
\hline & & & & & $2.7 \pm 0.7$ & $5.9 \pm 1.8$ & $0.140 \pm 0.011$ & $3.96 \pm 1.23^{2}$ \\
\hline & & & & & - & $3.2 \pm 1.0$ & $0.117 \pm 0.049$ & $1.78 \pm 1.21^{2}$ \\
\hline
\end{tabular}

Table 4 continued 
Table 4 (continued)

\begin{tabular}{|c|c|c|c|c|c|c|c|c|}
\hline Transition & $\begin{array}{c}\text { Wavenumber } \\
\left(\mathrm{cm}^{-1}\right)\end{array}$ & $\begin{array}{c}E_{l} / k_{b} \\
(\mathrm{~K})\end{array}$ & $g_{l}$ & $\begin{array}{c}A \\
\left(\mathrm{~s}^{-1}\right)\end{array}$ & $\begin{array}{c}v_{\mathrm{LSR}} \\
\left(\mathrm{km} \mathrm{s}^{-1}\right)\end{array}$ & $\begin{array}{c}v_{\mathrm{FWHM}} \\
\left(\mathrm{km} \mathrm{s}^{-1}\right)\end{array}$ & $\tau_{0}$ & $\begin{array}{c}N_{l} \\
\times 10^{14} \mathrm{~cm}^{-2}\end{array}$ \\
\hline \multirow[t]{2}{*}{ Q13e } & 713.304602 & 386.9 & 162 & 2.026 & $-7.3 \pm 0.2$ & $7.2 \pm 0.3$ & $0.419 \pm 0.015$ & $14.52 \pm 0.99^{1}$ \\
\hline & & & & & $-0.1 \pm 0.7$ & $7.6 \pm 1.0$ & $0.134 \pm 0.013$ & $4.87 \pm 1.00^{2}$ \\
\hline \multirow[t]{3}{*}{ Q14e } & 713.5078 & 446.5 & 174 & 2.026 & $-7.8 \pm 0.2$ & $6.1 \pm 0.3$ & $0.309 \pm 0.010$ & $9.08 \pm 0.65^{1}$ \\
\hline & & & & & $0.2 \pm 0.7$ & $8.0 \pm 1.2$ & $0.109 \pm 0.007$ & $4.18 \pm 0.81^{2}$ \\
\hline & & & & & - & $3.2 \pm 0.8$ & $0.086 \pm 0.032$ & $1.33 \pm 0.79^{2}$ \\
\hline \multirow[t]{2}{*}{$\mathrm{R} 0 \mathrm{e}$} & 714.935627 & 0.0 & 6 & 1.371 & $-6.7 \pm 0.3$ & $9.7 \pm 0.5$ & $0.438 \pm 0.011$ & $10.12 \pm 0.63^{1}$ \\
\hline & & & & & $2.4 \pm 0.8$ & $8.0 \pm 1.4$ & $0.112 \pm 0.014$ & $2.13 \pm 0.57^{2}$ \\
\hline \multirow[t]{2}{*}{ R1e } & 717.89124 & 4.3 & 18 & 1.251 & $-7.2 \pm 0.1$ & $8.5 \pm 0.1$ & $0.700 \pm 0.007$ & $28.23 \pm 0.54^{1}$ \\
\hline & & & & & $1.4 \pm 0.2$ & $6.5 \pm 0.4$ & $0.152 \pm 0.007$ & $4.66 \pm 0.40^{2}$ \\
\hline \multirow[t]{2}{*}{ R3e } & 723.800848 & 25.5 & 42 & 1.19 & $-7.2 \pm 0.1$ & $9.4 \pm 0.3$ & $0.779 \pm 0.019$ & $48.37 \pm 2.25^{1}$ \\
\hline & & & & & $2.1 \pm 0.3$ & $7.6 \pm 0.7$ & $0.212 \pm 0.018$ & $10.63 \pm 1.85^{2}$ \\
\hline \multirow[t]{2}{*}{$\mathrm{R} 4 \mathrm{e}$} & 726.7547 & 42.5 & 54 & 1.184 & $-7.3 \pm 0.1$ & $8.7 \pm 0.2$ & $0.780 \pm 0.013$ & $47.97 \pm 1.81^{1}$ \\
\hline & & & & & $1.1 \pm 0.6$ & $7.5 \pm 1.1$ & $0.154 \pm 0.014$ & $8.20 \pm 1.69^{2}$ \\
\hline R5e & 729.70782 & 63.8 & 66 & 1.183 & $-7.3 \pm 0.1$ & $11.6 \pm 0.2$ & $0.755 \pm 0.013$ & $65.02 \pm 1.25^{1}$ \\
\hline \multirow[t]{3}{*}{ R6e } & 732.660136 & 89.3 & 78 & 1.187 & $-7.2 \pm 0.2$ & $6.7 \pm 0.8$ & $0.698 \pm 0.091$ & $35.76 \pm 8.44^{1}$ \\
\hline & & & & & $-1.0 \pm 1.4$ & $11.0 \pm 1.6$ & $0.272 \pm 0.042$ & $22.99 \pm 6.66^{2}$ \\
\hline & & & & & - & $3.5 \pm 0.7$ & $0.183 \pm 0.073$ & $4.9^{r} \pm 2.84^{1}$ \\
\hline \multirow[t]{2}{*}{ R7e } & 735.611573 & 119.1 & 90 & 1.194 & $-7.5 \pm 0.2$ & $8.3 \pm 0.2$ & $0.760 \pm 0.018$ & $49.65 \pm 2.16^{1}$ \\
\hline & & & & & $0.9 \pm 0.7$ & $8.9 \pm 0.9$ & $0.183 \pm 0.014$ & $12.83 \pm 2.13^{2}$ \\
\hline \multirow[t]{2}{*}{ R10e } & 744.459871 & 233.9 & 126 & 1.221 & $-7.9 \pm 0.2$ & $8.1 \pm 0.4$ & $0.574 \pm 0.017$ & $38.59 \pm 2.37^{1}$ \\
\hline & & & & & $0.5 \pm 0.6$ & $7.9 \pm 0.9$ & $0.208 \pm 0.017$ & $13.56 \pm 2.32^{2}$ \\
\hline \multirow[t]{2}{*}{ R11e } & 747.407049 & 280.7 & 138 & 1.231 & $-7.3 \pm 0.3$ & $9.9 \pm 0.5$ & $0.468 \pm 0.013$ & $38.86 \pm 2.47^{1}$ \\
\hline & & & & & $1.4 \pm 0.7$ & $7.9 \pm 1.1$ & $0.138 \pm 0.021$ & $9.12 \pm 2.33^{2}$ \\
\hline R12e & 750.352972 & 331.7 & 150 & 1.242 & $-7.5 \pm 0.1$ & $7.9 \pm 0.3$ & $0.420 \pm 0.015$ & $28.00 \pm 1.26^{1}$ \\
\hline \multirow[t]{2}{*}{ R13e } & 753.297564 & 386.9 & 162 & 1.254 & $-7.9 \pm 0.3$ & $6.0 \pm 0.7$ & $0.247 \pm 0.046$ & $12.54 \pm 3.54^{1}$ \\
\hline & & & & & $-0.5 \pm 1.6$ & $12.0 \pm 3.0$ & $0.139 \pm 0.015$ & $14.14 \pm 4.73^{2}$ \\
\hline \multirow[t]{2}{*}{$\mathrm{R} 15 \mathrm{e}$} & 759.182446 & 510.2 & 186 & 1.278 & $-7.3 \pm 0.6$ & $7.6 \pm 1.0$ & $0.143 \pm 0.011$ & $9.31 \pm 1.58^{1}$ \\
\hline & & & & & $1.3 \pm 0.8$ & $8.4 \pm 1.4$ & $0.108 \pm 0.009$ & $7.76 \pm 1.62^{2}$ \\
\hline R17e & 765.061068 & 650.4 & 210 & 1.302 & $-1.4 \pm 0.7$ & $15.4 \pm 2.5$ & $0.051 \pm 0.006$ & $6.77 \pm 1.40^{\circ}$ \\
\hline R18e & 767.997835 & 726.9 & 222 & 1.315 & $-0.7 \pm 0.5$ & $7.9 \pm 1.9$ & $0.068 \pm 0.012$ & $4.70 \pm 1.57^{0}$ \\
\hline
\end{tabular}

Note-See Table 2 for column descriptions. Data in the first five columns are from the HITRAN database (Gordon et al. 2017). Superscripts in the final column refer to which velocity component $N_{l}$ will be totalled towards for the rotation diagrams in $\S 3.4:{ }^{0}$ none, ${ }^{1}$ primary, ${ }^{2}$ secondary. Velocity for lines with more than one Gaussian is taken to be the that of the line with the largest $\tau_{0}$ and the other Gaussian is recorded without velocity and in italics.

The LSR velocities of $\mathrm{HNC}, \mathrm{H}^{13} \mathrm{CN}$, and the primary component of $\mathrm{HCN}$ fall in the range of $\sim-6$ to $-8 \mathrm{~km} \mathrm{~s}^{-1}$, making it highly likely that they trace the same component in IRc2. The secondary HCN velocity component is $\sim 1 \mathrm{~km} \mathrm{~s}^{-1}$. The line widths $\left(v_{\mathrm{FWHM}}=2 \sqrt{2 \ln 2} \sigma_{v}\right)$ of $\mathrm{HNC}$ are systematically higher than the primary component of HCN by about $3 \mathrm{~km} \mathrm{~s}^{-1}$. Furthermore, Rangwala et al. (2018) observing the same $\mathrm{HCN}$ transitions as the present work, only resolve a sin- gle $\mathrm{HCN}$ velocity component with $v_{\mathrm{FWHM}}$ between 9 to $14 \mathrm{~km} \mathrm{~s}^{-1}$, which is comparable to the HNC line widths in Tabe 2. Clearly, HNC may also have an unresolved, secondary velocity component.

Two HCN lines, R17e and R18e, are not counted towards any component, due to high noise and LSR velocities of $\sim-1 \mathrm{~km} \mathrm{~s}^{-1}$, which do not fit with either component. These lines were not resolved enough for a fit consistent with the other lines, but we include their de- 
rived parameters nonetheless in Table 4 for completeness as they are clearly detected in Rangwala et al. (2018).

\subsection{Rotation Diagrams}

For molecular populations in local thermal equilibrium (LTE), the level populations follow a Boltzman distribution (Goldsmith \& Langer 1999) given by:

$$
\ln \frac{N_{l}}{g_{l}}=\ln \frac{N}{Q_{R}\left(T_{e x}\right)}-\frac{E_{l}}{k_{b} T_{e x}}
$$

where $N$ is the total column density, $Q_{R}$ is the rotational partition function, $E_{l}$ is the energy level of the lower state, and $T_{e x}$ is the excitation temperature. We calculated $Q_{R}$ for $\mathrm{HCN}$ and $\mathrm{H}^{13} \mathrm{CN}$ for a given $T_{e x}$ with the HITRAN python interface HAPI (Kochanov et al. 2016) and for HNC from levels published in ExoMol (Harris et al. 2006; Barber et al. 2013).

Figure 4 gives the rotation diagrams for $\mathrm{HNC}, \mathrm{H}^{13} \mathrm{CN}$, and the two velocity components of HCN. This shows the linear relationship between $\ln \left(N_{l} / g_{l}\right)$ and $E_{l} / k_{b}$, implying that the LTE approximation holds. By linearly fitting to Equation 6, we obtain the values of $T_{e x}$ and $N$ for these species and components, summarized in Table 5 along with their abundance relative to $\mathrm{H}_{2}$.

Several observations give $N_{\mathrm{H}_{2}} \sim 10^{23}$ to $5 \times 10^{24} \mathrm{~cm}^{-2}$ towards IRc2 (e.g., Evans et al. 1991; Schilke et al. 1992; Sutton et al. 1995; Persson et al. 2007; Tercero et al. 2010; Favre et al. 2011; Plume et al. 2012; Crockett et al. 2014; Feng et al. 2015) and we adopt the most recent calculation derived from ALMA dust continuum emission, $N_{\mathrm{H}_{2}}=(4.7 \pm 0.2) \times 10^{24} \mathrm{~cm}^{-2}$ (Peng et al. 2019), as it has a comparable beam size to SOFIA/EXES. However, uncertainties stand with adopting this value because the $\mathrm{H}_{2}$ distribution may differ from the that of the larger molecules HCN and HNC, and these observations may correspond to a different component of the ISM towards IRc2. We do not fit the $\mathrm{P}$ branch of $\mathrm{HCN}$, given only two points for the primary component and one for the secondary. Most of the $\mathrm{P}$ branch lines fell between observed settings.

Both $\mathrm{HNC}$ and $\mathrm{H}^{13} \mathrm{CN}$ trace the coldest gas at $\sim 100$ $\mathrm{K}$, while $\mathrm{HNC}$ has a lower column density at $7.7 \times 10^{14}$ $\mathrm{cm}^{-2}$ compared to $4.4 \times 10^{15} \mathrm{~cm}^{-2}$ for $\mathrm{H}^{13} \mathrm{CN}$. $\mathrm{HCN}$ traces the hottest, highest column density gas. Its primary velocity component (Q: $177 \mathrm{~K}$; R: $153 \mathrm{~K}$ ) is cooler than its secondary component (Q: $348 \mathrm{~K}$; R: $270 \mathrm{~K}$ ). However, the primary component has a higher column density (Q: $4.5 \times 10^{16} \mathrm{~cm}^{-2}$; R: $6.5 \times 10^{16} \mathrm{~cm}^{-2}$ ) compared to the secondary in both branches $\left(\mathrm{Q}: 1.1 \times 10^{16}\right.$ $\mathrm{cm}^{-2}$; R: $\left.2.1 \times 10^{16} \mathrm{~cm}^{-2}\right)$.

\section{DISCUSSION}

\subsection{Interpretation of Results}

The $-7 \mathrm{~km} \mathrm{~s}^{-1}$ component of $\mathrm{HNC}, \mathrm{HCN}$, and $\mathrm{H}^{13} \mathrm{CN}$ has also been observed in the MIR by Rangwala et al. (2018) and Lacy et al. (2005) for $\mathrm{HCN}$ and $\mathrm{C}_{2} \mathrm{H}_{2}$, blueshifted with respect to the ambient cloud velocity of $9 \mathrm{~km} \mathrm{~s}^{-1}$ (Zapata et al. 2012). As discussed in Rangwala et al. (2018), this velocity is similar to the North-East segment of the bipolar outflow originating from radio source I, which has been identified and mapped with $\mathrm{SiO}$ and $\mathrm{H}_{2} \mathrm{O}$ masers (Genzel et al. 1981; Wright et al. 1995; Plambeck et al. 2009). Our $-7 \mathrm{~km} \mathrm{~s}^{-1}$ lines may be associated with this outflow, being expelled material irradiated by IRc2, while the $1 \mathrm{~km} \mathrm{~s}^{-1}$, hotter component is very likely closer to the hot core itself. Though we only resolved the $1 \mathrm{~km} \mathrm{~s}^{-1}$ component for HCN, the FWHMs of HNC in Table 2 are systematically larger than the HCN lines in Table 4. It may be that HNC also has a $1 \mathrm{~km} \mathrm{~s}^{-1}$ component that is unresolved by our observations. Our $\mathrm{H}^{13} \mathrm{CN}$ lines are too weak for this analysis.

The HNC column densities in Table 5 are consistently lower than HCN by about two orders of magnitude, evidence for a highly irradiated environment. Indeed, studies of planetary nebulae and the Helix Nebula find that $\mathrm{HCN} / \mathrm{HNC}$ correlates with increasing UV radiation (Bublitz et al. 2019, 2020). HNC is less photostable than HCN (Chenel et al. 2016; Aguado et al. 2017) and is destroyed in increasingly advanced and hotter stages of stellar evolution (Jin et al. 2015). We see that towards Orion IRc2, HCN is more abundant and at hotter temperatures compared to $\mathrm{HNC}$, in support of these findings.

A similar high resolution ( 55,000 to 85,000) MIR spectral survey covered the hot cores AFGL 2591 and AFGL 2136 from 4 to $13 \mu \mathrm{m}$ with SOFIA/EXES, and the TEXES and the iSHELL instruments on the NASA Infrared Telescope Facility (Barr et al. 2020). In both hot cores, they identified transitions from the $\mathrm{HCN} \nu_{2}$ band, $\mathrm{R}$ branch that are also present in our Orion IRc2 survey. Their rotation diagrams for HCN yield temperatures $674.8 \pm 32.0 \mathrm{~K}$ and $624.6 \pm 19 \mathrm{~K}$ for AFGL 2591 and AFGL 2136 respectively. This is much hotter than the $\mathrm{R}$ branch temperatures for IRc2 that we find, $153 \pm 9$ $\mathrm{K}$ and $270 \pm 32 \mathrm{~K}$ for each velocity component. IRc2 may be cooler than these two conventional hot cores due to its lack of an internal heat source. Similar to our conclusion, Barr et al. (2020) emphasize that the MIR probes hot gas at the centre of hot cores, compared to the sub-mm that probes the outer envelope. 

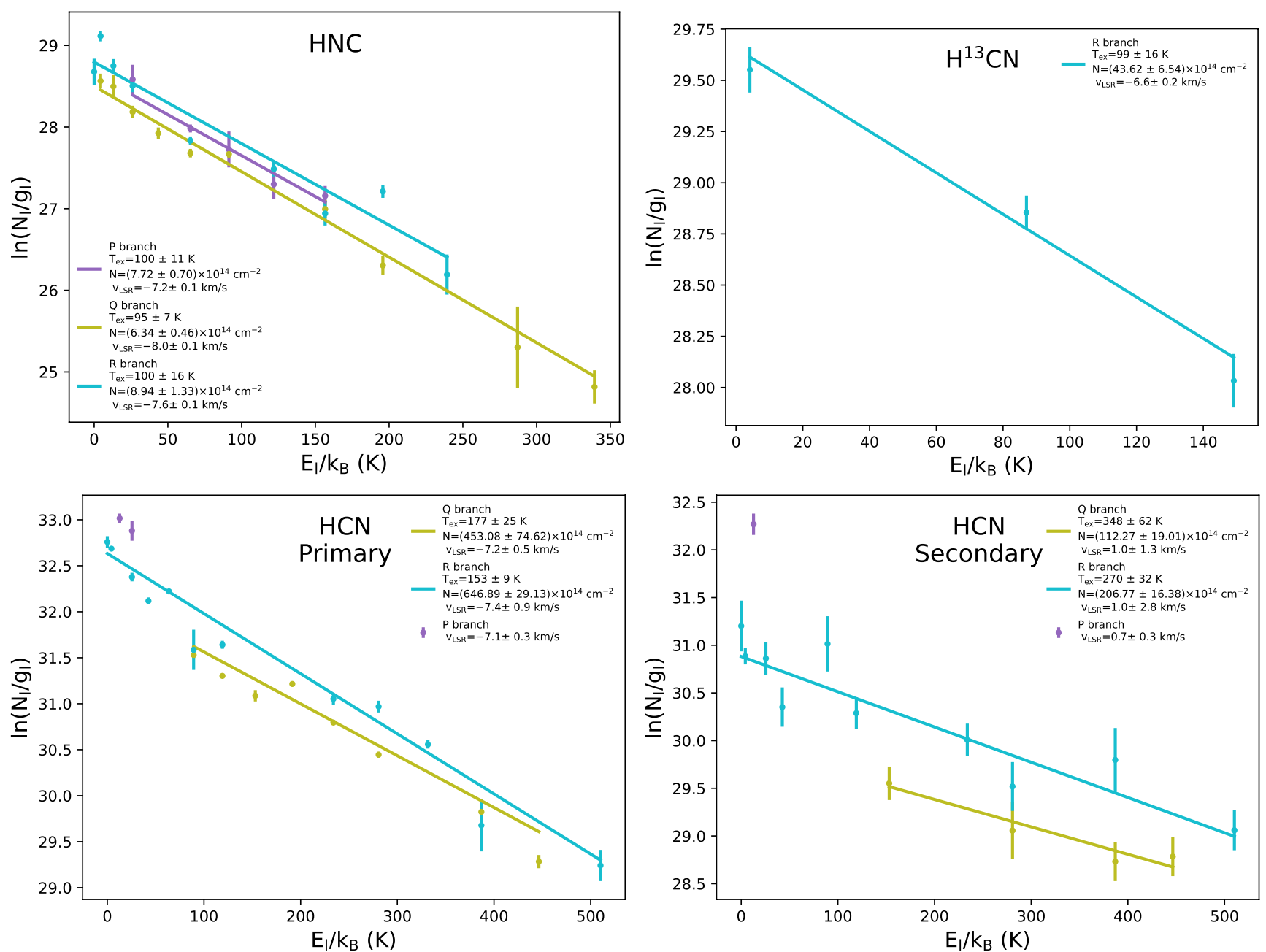

Figure 4. Rotation diagrams for $\mathrm{HNC}$ (top left), $\mathrm{H}^{13} \mathrm{CN}$ (top right), HCN primary velocity component (bottom left), and HCN secondary velocity component (bottom right). These follow Equation 6 where the excitation temperature and total column density of each species is extracted from the relation between the energy levels, column densities, and lower statistical weights of the transitions. The average LSR velocity for each branch is also given.

\subsection{Comparison to Previous IRc2 Observations}

Table 6 summarizes derived velocities, temperatures, and column densities for $\mathrm{HNC}, \mathrm{HCN}$, and $\mathrm{H}^{13} \mathrm{CN}$ for observations towards Orion IRc2 from the literature and compares these to the present study. We take the average across all branches from Table 5 for each component to display in Table 6 . The most apparent difference is that the two spectral regimes reveal different components towards Orion IRc2. The sub- $\mathrm{mm} / \mathrm{mm}$ observations detect these three species in emission with a positive $v_{\text {LSR }}$ while the MIR observations detect them in absorption with a negative $v_{\mathrm{LSR}}$. Another difference is that all observations in the sub- $\mathrm{mm} / \mathrm{mm}$ and MIR with ISO (Boonman et al. 2003) have a much larger beam size than EXES (Rangwala et al. 2018) and TEXES (Lacy et al. 2002; Lacy et al. 2005). The smaller beam is important to observing the ISM component closest to the hot core. However, previous MIR observations do not resolve the secondary $\mathrm{HCN}$ velocity component present in our work (Lacy et al. 2005; Rangwala et al. 2018). Our MIR observations are able to probe closer to the host core itself, and find the hottest measured temperature for HCN towards Orion IRc2 to date.

Table 7 compares $\mathrm{HCN} / \mathrm{HNC}$ and ${ }^{12} \mathrm{C} /{ }^{13} \mathrm{C}$ ratios from this work to the literature. We use the average values over all branches of $\mathrm{HCN}, \mathrm{HNC}$, and $\mathrm{H}^{13} \mathrm{CN}$ from Table 1 for these ratios, taking only the $-7 \mathrm{~km} \mathrm{~s}^{-1}$ component of $\mathrm{HCN}$, to find $\mathrm{HCN} / \mathrm{HNC}=72 \pm 7$ and ${ }^{12} \mathrm{C} /{ }^{13} \mathrm{C}=13 \pm 2$.

Our ratio $\mathrm{HCN} / \mathrm{HNC}=72 \pm 7$ falls within the the lower end of previous measurements that range from 75 to 300 (Goldsmith et al. 1986; Schilke et al. 1992; Comito et al. 2005). Such a range of values makes it difficult to draw direct a comparison between observations. Widely differing beam sizes may be responsible, where ours is 
Table 5. Overview of Species Properties

\begin{tabular}{ccccc}
\hline \hline Branch & $\begin{array}{c}v_{\mathrm{LSR}} \\
\left(\mathrm{km} \mathrm{s}^{-1}\right)\end{array}$ & $\begin{array}{c}T_{\mathrm{ex}} \\
(\mathrm{K})\end{array}$ & $\times 10^{14} \mathrm{~cm}^{-2}$ & $\begin{array}{c}N / N_{\mathrm{H}_{2}} \\
\times 10^{-9}\end{array}$ \\
\hline \multicolumn{5}{c}{$\mathrm{HNC}$} \\
$\mathrm{P}$ & $-7.2 \pm 0.1$ & $100 \pm 11$ & $7.72 \pm 0.70$ & $0.16 \pm 0.02$ \\
$\mathrm{Q}$ & $-8.0 \pm 0.1$ & $95 \pm 7$ & $6.34 \pm 0.46$ & $0.13 \pm 0.01$ \\
$\mathrm{R}$ & $-7.6 \pm 0.1$ & $100 \pm 16$ & $8.94 \pm 1.33$ & $0.19 \pm 0.03$ \\
\hline \multicolumn{5}{c}{$\mathrm{HCN}$} \\
$\mathrm{P}$ & $-7.1 \pm 0.3$ & - & - & - \\
$\mathrm{Q}$ & $0.7 \pm 0.3$ & - & - & - \\
& $-7.2 \pm 0.5$ & $177 \pm 25$ & $453.08 \pm 74.62$ & $9.64 \pm 1.64$ \\
$\mathrm{R}$ & $-7.0 \pm 1.3$ & $348 \pm 62$ & $112.27 \pm 19.01$ & $2.39 \pm 0.42$ \\
& $1.0 \pm 2.8$ & $270 \pm 32$ & $206.77 \pm 16.38$ & $4.40 \pm 0.40$ \\
\hline \multicolumn{5}{c}{$\mathrm{H}^{13} \mathrm{CN}$} \\
\hline $\mathrm{R}$ & $-6.6 \pm 0.2$ & $99 \pm 16$ & $43.62 \pm 6.54$ & $0.93 \pm 0.14$ \\
\hline
\end{tabular}

Note-For each species, branch, and velocity component, $v_{\text {LSR }}$ is the average local standard rest of velocity, $T_{e x}$ is the excitation temperature, $N$ is the total column density, and $N_{\mathrm{H}_{2}}$ is the column density of $\mathrm{H}_{2}$, adopted to be $4.7 \pm 0.2 \times 10^{24} \mathrm{~cm}^{-2}$ (Peng et al. 2019). The HCN P branch did not have enough points for a linear fit to find temperature and column density.

the smallest at $3{ }^{\prime \prime} 2$, and others are: $11^{\prime \prime}$ (Comito et al. 2005), 13 to $26^{\prime \prime}$ (Schilke et al. 1992), and 42 to $60^{\prime \prime}$ (Goldsmith et al. 1986). We should also note that we measure a different velocity components than the sub$\mathrm{mm} / \mathrm{mm}$ studies, and that there are no previous studies in the MIR with which to compare our results directly. Our smaller beam size allows us to probe the hot core more closely.

The ${ }^{12} \mathrm{C} /{ }^{13} \mathrm{C}=13 \pm 2$ ratio towards IRc2 has been measured by more studies. Because we only saw three lines of $\mathrm{H}^{13} \mathrm{CN}$, the uncertainty in the ratio may be larger than quoted. With SOFIA/EXES MIR measurements of $\mathrm{C}_{2} \mathrm{H}_{2}$, Rangwala et al. (2018) find ${ }^{12} \mathrm{C} /{ }^{13} \mathrm{C}=$ $14 \pm 1$, similar to our value despite the difference in species to obtain it. Both these values are close to the Tercero et al. (2010) estimation for the hot core using $\mathrm{H}_{2} \mathrm{CS}, 20 \pm 9$, but are much lower than other measurements that range from 53 to 82.6 (Schilke et al. 1997; Favre et al. 2014; Feng et al. 2015), using HCN, $\mathrm{HCOOCH}_{3}$, and $\mathrm{CH}_{3} \mathrm{CN}$ respectively.

With Orion's distance from the Galactic Center and a linear relation derived from Milam et al. (2005), Favre et al. (2014) calculated that the expected ratio is 50 to 90. This value is similar to measurements towards the neighboring compact ridge from 30 to 80 (Blake et al.
1987; Persson et al. 2007; Gong et al. 2015). One explanation for the lower ratio we and Rangwala et al. (2018) find towards the hot core is that previous studies conducted in the sub- $\mathrm{mm} / \mathrm{mm}$ and radio wavelengths observe different components than we do in the MIR, as illustrated in Table 6. As our lines are optically thin, then there must be either a physical or chemical explanation for this low ratio.

Our ${ }^{12} \mathrm{C} /{ }^{13} \mathrm{C}$ is comparable to the ratio towards the Galactic Central hot core Sgr B2, 20 (Favre et al. 2014; Giesen et al. 2020), which is expected for the region (Milam et al. 2005; Yan et al. 2019). Several measurements have found the ratio to be lower in star-forming regions than in the local ISM: 20-50 (Daniel et al. 2013), 30 towards a protostellar binary (Jørgensen et al. 2018), $45 \pm 3$,(Magalhães et al. 2018), and $\sim 16$ (Bøgelund et al. 2019) for various molecules. Colzi et al. (2020) suggest that an exchange reaction between ${ }^{13} \mathrm{C}$ and $\mathrm{C}_{3}$ can lead to a ${ }^{13} \mathrm{C}$ enhancement in molecular clouds while they are still $<30 \mathrm{~K}$. This is cooler than our excitation temperatures for all species in Table 1. In this case, our low ratio may reflect conditions prior to protostellar warmup. 
Table 6. Comparison of HNC, HCN, and $\mathrm{H}^{13} \mathrm{CN}$ observations towards Orion IRc2 between this and previously published works.

\begin{tabular}{|c|c|c|c|c|c|c|c|}
\hline Reference & Region & Beam Size & $\tau$ & Type & $\begin{array}{c}v_{\mathrm{LSR}} \\
\left(\mathrm{km} \mathrm{s}^{-1}\right)\end{array}$ & $\begin{array}{c}T \\
(\mathrm{~K})\end{array}$ & $\begin{array}{c}N \\
\left(\times 10^{14} \mathrm{~cm}^{-2}\right)\end{array}$ \\
\hline \multicolumn{8}{|c|}{$\mathrm{HNC}$} \\
\hline This work & MIR & $5 .^{\prime \prime} 5-6^{\prime \prime} 9 \times 3^{\prime \prime} .2$ & thin & abs & $-7.6 \pm 0.1$ & $98 \pm 7$ & $7.67 \pm 0.52$ \\
\hline Persson et al. (2007) & sub-mm & 2.1 & thin & emi & 9 & - & 4.4 \\
\hline Comito et al. (2005) & sub-mm & $11^{\prime \prime}$ & thin & emi & 8 & $150^{a}$ & 5 \\
\hline \multicolumn{8}{|c|}{$\mathrm{HCN}$} \\
\hline \multirow[t]{2}{*}{ This work } & \multirow[t]{2}{*}{ MIR } & \multirow[t]{2}{*}{$1^{\prime \prime} 9-2^{\prime \prime} .1 \times 3^{\prime \prime} .2$} & \multirow[t]{2}{*}{ thin } & \multirow[t]{2}{*}{ abs } & $-7.3 \pm 0.5$ & $165 \pm 13$ & $549.99 \pm 40.05$ \\
\hline & & & & & $1.0 \pm 1.5$ & $309 \pm 35$ & $159.52 \pm 12.55$ \\
\hline Rangwala et al. (2018) & MIR & $3^{\prime \prime} .2 \times 3^{\prime \prime} .2$ & thin & abs & $-5.2 \pm 2.8$ & $140 \pm 10^{b}$ & $840 \pm 60$ \\
\hline Comito et al. (2005) & sub-mm & $11^{\prime \prime}$ & thin & emi & 5 & $150^{a}$ & 700 \\
\hline Lacy et al. (2005) & MIR & $1^{\prime \prime} 5 \times 8^{\prime \prime} c$ & $-c$ & abs & $-10^{d}$ & $150^{d}$ & - \\
\hline Boonman et al. (2003) & MIR & $14^{\prime \prime} \times 27^{\prime \prime}$ & thick & abs & - & $275^{e}$ & 450 \\
\hline Schilke et al. (2001) & sub-mm & $12^{\prime \prime}$ & thin & emi & - & $100^{f}$ & 260 \\
\hline Stutzki et al. (1988) & sub-mm & $32^{\prime \prime}$ & thick & emi & 6 & - & - \\
\hline Blake et al. (1987) & $\mathrm{mm}$ & $30^{\prime \prime}$ & thick & emi & 5.8 & - & 250 \\
\hline \multicolumn{8}{|c|}{$\mathrm{H}^{13} \mathrm{CN}$} \\
\hline This work & MIR & $2^{\prime \prime} 1 \times 3^{\prime \prime} .2$ & thin & abs & $-6.6 \pm 0.2$ & $97 \pm 13$ & $42.08 \pm 5.67$ \\
\hline Schilke et al. (1992) & $\mathrm{mm}$ & $26^{\prime \prime}$ & thin & emi & $9.2^{g}$ & $70^{g}$ & 0.57 \\
\hline
\end{tabular}

Note-Left to right, columns refer to observation reference, spectral region, beam size, whether the observation is optically thin or thick, whether the line type is emission (emi) or absorption (abs), LSR velocity, temperature, and column density. Temperatures are calculated by a variety of methods, as detained in table footnotes. The HNC and HCN values in this work are the averages across all branches for each component from Table 5. For reference, the ambient cloud velocity is $9 \mathrm{~km} \mathrm{~s}^{-1}$ (Zapata et al. 2012).

$a_{\text {rotation temperature derived from DCN }}$

$b$ excitation temperature, $\mathrm{R}$ branch only

${ }^{c}$ beam size from Lacy et al. (2002), opacity unknown

$d_{v_{\mathrm{LSR}}}$ and temperature approximation from $\mathrm{HCN}$ and $\mathrm{C}_{2} \mathrm{H}_{2}$ lines

$e_{\text {modelled excitation temperature }}$

$f_{\text {rotation temperature derived from } \mathrm{H}^{15} \mathrm{CN}}$

$g_{v_{\text {LSR }}}$ and kinetic temperature estimated for entire hot core

\subsection{Comparison to a Hot Core Model}

To compare our observations to modelling, we utilized the gas-grain chemical network as described in Acharyya \& Herbst (2018) for the model in Figures 5 and 6. Both the gas-phase and grain-surface chemistry are treated via a rate equation approach. The major features of the model are as follows:

- Model runs have two physical evolutionary phases as prescribed by Brown et al. (1988). In the first phase, free fall collapse (Figure 5), the cloud undergoes isothermal collapse at $10 \mathrm{~K}$, from a density of 3,000 to $10^{7} \mathrm{~cm}^{-3}$ in just under $10^{6}$ years. During this period the visual extinction grows from 1.64 to $432 \mathrm{mag}$. In the second phase, warmup (Figure 6, left column), the collapse stops and the temperature increases linearly at the rate of $1 \mathrm{~K} / 250$ years, which is a representative heating rate for high-mass star-formation. We ran warmup models for five different final temperatures: 100, 150, 200, 270 and $300 \mathrm{~K}$. Once the hot core reaches its final temperature, its chemical evolution continues through the third phase, post-warmup, until $10^{7}$ years elapse (Figure 6, right column). 
Table 7. Comparison of observed ratios towards Orion IRc2

\begin{tabular}{ccc}
\hline \hline Reference & Spectral Region & Ratio \\
\hline \multicolumn{3}{c}{ HCN/HNC } \\
\hline This work & MIR & $72 \pm 7$ \\
Comito et al. (2005) & sub-mm & 140 \\
Schilke et al. (1992) & $\mathrm{mm}$ & $80 \pm 5$ \\
Goldsmith et al. (1986) & $\mathrm{mm}$ & $\sim 200-300$ \\
\hline \multicolumn{3}{c}{${ }^{12} \mathrm{C} /{ }^{13} \mathrm{C}$} \\
This work & $\mathrm{MIR}$ & $13 \pm 2^{a}$ \\
Rangwala et al. (2018) & $\mathrm{MIR}$ & $14 \pm 1^{b}$ \\
Feng et al. (2015) & sub-mm & $79.6 \pm 3.0^{c}$ \\
Favre et al. (2014) & $\mathrm{mm}$ & $\geq 53^{d}$ \\
Tercero et al. (2010) & $\mathrm{mm}$ & $20 \pm 9^{e}$ \\
Schilke et al. (1997) & radio & $60^{a}$ \\
\hline
\end{tabular}

Note- Species used to obtain ${ }^{12} \mathrm{C} /{ }^{13} \mathrm{C}$ ratio:

$a_{\mathrm{HCN}}$

${ }^{b} \mathrm{C}_{2} \mathrm{H}_{2}$

${ }^{c} \mathrm{CH}_{3} \mathrm{CN}$

$d_{\mathrm{HCOOCH}_{3}}$

${ }^{e} \mathrm{H}_{2} \mathrm{CS}$

- Graninger et al. (2014) found that the reaction $\mathrm{HNC}+\mathrm{H} \rightarrow \mathrm{HCN}+\mathrm{H}$ regulates the $\mathrm{HCN} / \mathrm{HNC}$ ratio under all conditions. However, the activation barrier of this reaction is highly uncertain, and can vary between 0 and 2,000 K. Therefore, we study the cases with and without the $2,000 \mathrm{~K}$ activation barrier.

- Classical dust grains are $0.1 \mu \mathrm{m}$ in size with a surface site density $n_{\mathrm{s}}=1.5 \times 10^{15} \mathrm{~cm}^{-2}$, leading to about $10^{6}$ adsorption binding sites per grain.

- We use standard low-metal elemental abundances, initially in the form of gaseous atoms with the exception of $\mathrm{H}_{2}$. Elements with ionization potentials below $13.6 \mathrm{eV}$ take the form of singly charged positive ions $\left(\mathrm{C}^{+}, \mathrm{Fe}^{+}, \mathrm{Na}^{+}, \mathrm{Mg}^{+}, \mathrm{S}^{+}, \mathrm{Si}^{+}\right.$, and $\left.\mathrm{Cl}^{+}\right)$.

- We use a sticking coefficient of 1 , standard cosmicray ionisation rate of $10^{-17} \mathrm{~s}^{-1}$, and a diffusionto-binding energy ratio of 0.5 .

Figure 5 shows the time variation of $\mathrm{HCN}$ and $\mathrm{HNC}$ abundances during the freefall collapse phase during which the temperature is constant at $10 \mathrm{~K}$. The peak abundances (relative to total atmoic hydrogen) for $\mathrm{HCN}$

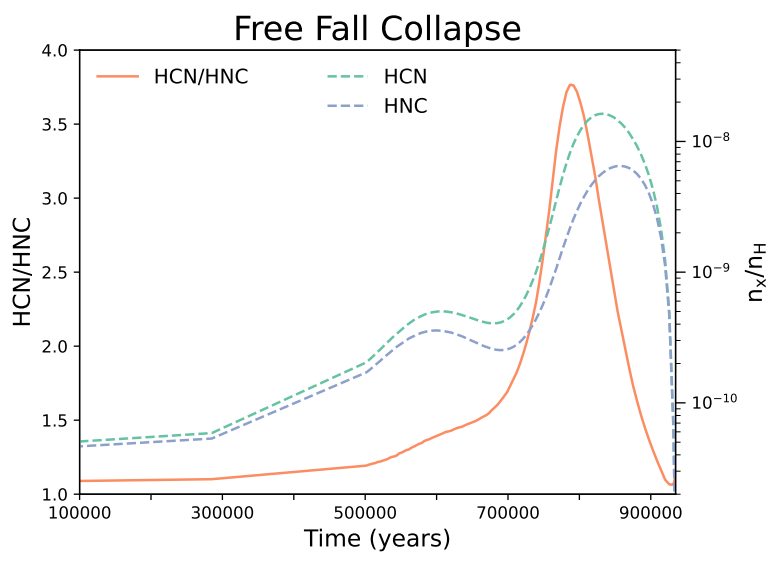

Figure 5. Time variation of $\mathrm{HCN} / \mathrm{HNC}$ (orange solid line, left axis) and HCN and HNC abundances (green and blue dotted lines, right axis) during the free fall collapse phase of the model.

and $\mathrm{HNC}$ are $1.6 \times 10^{-8}$ and $6.5 \times 10^{-8}$ respectively. Towards the end of the first phase, $\mathrm{HCN}$ and HNC freeze out on the dust grains. The peak $\mathrm{HCN} / \mathrm{HNC}$ ratio is $\sim$ 3.8 .

Figure 6 shows the time variation of $\mathrm{HCN}$ and $\mathrm{HNC}$ for ten different models during the warmup and postwarmup phases, with five different maximum temperatures of 100, 150, 200, 270 and $330 \mathrm{~K}$, with or without the activation barrier. The left column of Figure 6 shows this chemical evolution during the warmup phase and the right column shows this in the post-warmup phase. Usually, the warmup phase lasts only up to $\sim 10^{4}$ years. However, we have plotted up to $10^{7}$ years to show the effect of a long lifetime.

It is clear that for $\mathrm{HCN}$ the abundances for all models are very similar until the warmup phase, with the exception of the $100 \mathrm{~K}$ model. However in the post-warmup phase, models deviate slightly depending on activation barrier, and varying temperature causes more deviation. For HNC, the deviation with and without the activation barrier begins in the warmup phase by a factor of 20 , and all ten models deviate the most during the post-warmup phase. Overall, the differences between models during the warmup phase are small, and they vary more during the post-warmup phase.

During post-warmup, the abundances for $\mathrm{HCN}$ in the 100 and $150 \mathrm{~K}$ models decreases by several orders of magnitude; whereas for the $200 \mathrm{~K}$ model, the reduction is about two orders of magnitude; and for the 270 and $330 \mathrm{~K}$ models, the change is minor. For HNC, a large reduction is present for all the models with no activation barrier. With a barrier, only the 100 and $150 \mathrm{~K}$ models show a large reduction. 

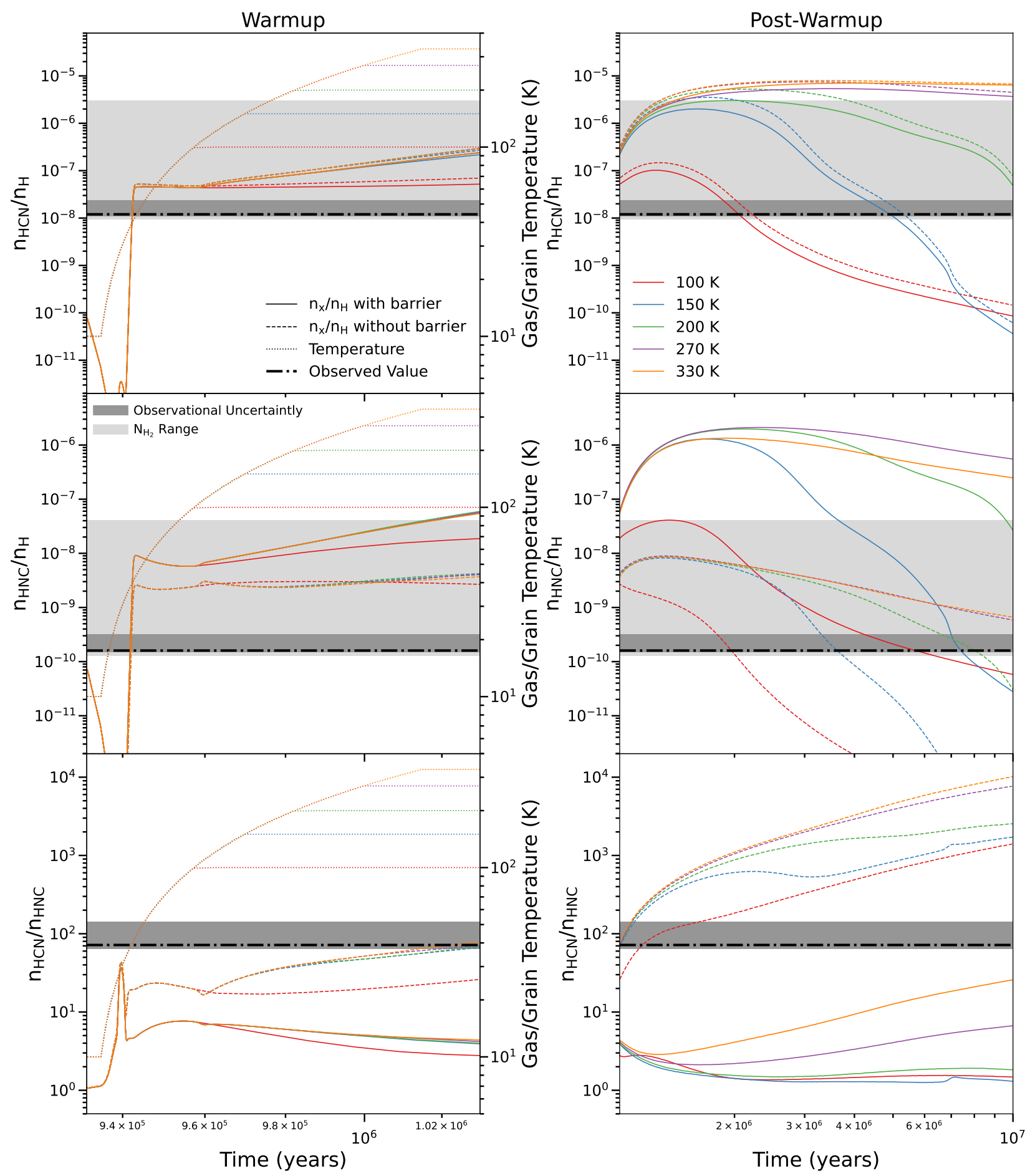

Figure 6. Time variation of HCN (top tow), HNC (middle row), and HCN/HNC (bottom row) during the warmup (left column) and post-warmup phase (right column) on the left $\mathrm{y}$-axes. Solid lines and dashed lines correspond to $\mathrm{HNC}+\mathrm{H} \rightarrow$ $\mathrm{HCN}+\mathrm{H}$ with and without a $2000 \mathrm{~K}$ barrier respectively. Time variation of gas/grain temperature is shown by dotted lines corresponding to the right y-axis during the warmup phase. Colour for all three line types corresponds to the final temperature reached during the warmup phase. The horizontal dash-dotted lines represent the observed values for $N_{\mathrm{HCN}} / N_{\mathrm{H}_{2}}, N_{\mathrm{HNC}} / N_{\mathrm{H}_{2}}$, and $N_{\mathrm{HCN}} / N_{\mathrm{HNC}}$ derived from Table 5 by taking the average across all branches for the $-7 \mathrm{~km} \mathrm{~s}^{-1}$ component and using our adopted $N_{\mathrm{H}_{2}}=(4.7 \pm 0.2) \times 10^{24} \mathrm{~cm}^{-2}$ (Peng et al. 2019). The dark grey box corresponds to the error. The light grey box reflects the extrema in the measured $\mathrm{N}_{\mathrm{H}_{2}}$ considering the lowest value $2 \times 10^{22} \mathrm{~cm}^{-2}$ from Persson et al. (2007) and the highest value of $5.4 \times 10^{24} \mathrm{~cm}^{-2}$ from Favre et al. (2011). Legends apply to all the panels. 
Figure 6 also includes lines for $N_{\mathrm{HCN}} / N_{\mathrm{H}_{2}}$, $N_{\mathrm{HNC}} / N_{\mathrm{H}_{2}}$, and $N_{\mathrm{HCN}} / N_{\mathrm{HNC}}$ as measured from Table 5 taking the average across all branches for the -7 $\mathrm{km} \mathrm{s}^{-1}$ velocity component. We do not display the 1 $\mathrm{km} \mathrm{s}^{-1}$ component of HCN given that there is no similar component in HNC. Our observed values for $N_{\mathrm{x}} / N_{\mathrm{H}_{2}}$ are comparable to the simulation's $n_{\mathrm{x}} / n_{\mathrm{H}}$ because in this situation we can assume that all hydrogen is molecular. We take $N_{\mathrm{H}_{2}}=(4.7 \pm 0.2) \times 10^{24} \mathrm{~cm}^{-2}$ (Peng et al. 2019) as our default value from the literature, given the similarity in the ALMA beam size to EXES. However, as discussed in $\S 3.4$, the observed $N_{\mathrm{H}_{2}}$ towards IRc2 varies greatly. We also take the extreme values from $2 \times 10^{22} \mathrm{~cm}^{-2}$ (Persson et al. 2007) to $5.4 \times 10^{24}$ $\mathrm{cm}^{-2}$ (Favre et al. 2011). This shows how much the uncertainty in our HCN and HNC column densities is dwarfed by the range of values that $N_{\mathrm{H}_{2}}$ takes in the literature. Because of this, $N_{\mathrm{HCN}} / N_{\mathrm{HNC}}$ offers a much more robust comparison to the model.

Indeed, our HCN and HNC abundances fall short of all ten models employed during the warmup phase. They coincide slightly for the $100 \mathrm{~K}$ no-barrier model at $2 \times 10^{6}$ years, as well as the $150 \mathrm{~K}$ barrier model at about $7 \times 10^{6}$ years. If we take into consideration, however, that our abundances are dependent on the widely varying $N_{\mathrm{H}_{2}}$, then it is impossible to match our data to any one model as they all fall within the uncertainties. We prefer instead to make our prediction based on $\mathrm{HCN} / \mathrm{HNC}$, which does not require the molecular hydrogen column density. In this situation, our observations match the 150 to $330 \mathrm{~K}$ no-barrier models at about $10^{6}$ years. This is similar to the hot core age Rangwala et al. (2018) derived by matching their observed $\mathrm{C}_{2} \mathrm{H}_{2}$ and HCN abundances to hot core models.

The OMC-1 region was the site of a powerful explosion about 500 years ago (Gómez et al. 2008). The $10^{6}$ years required to produce $\mathrm{HCN} / \mathrm{HNC}=72 \pm 7$ is much longer than the 500 year-old explosive event in the Orion Molecular Cloud. Given the lack of evidence for an embedded protostar that internally heats IRc2, there are two explanations for this mismatch in timescales. The first is that IRc2 may have been irradiated prior to the explosive event by proximity to radio source I and was later expelled. As such, it would have resembled a traditional, internally heated hot core prior to explosion.

The second possible explanation is that our modelling does not take shock physics into account, and the inclusion of such may alter the timescales needed to reach our $\mathrm{HCN} / \mathrm{HNC}$ ratio and our predicted age. Recent observations (Wright \& Plambeck 2017) suggest the region is indeed shocked. The competing role of C-type shocks in the Galactic Central hot core Sgr B2(N) has just been studied by Zhang et al. (2020). In addition, Willis et al. (2020) report new hot core models of Sgr B2(N) that use a single phase in which density and temperature are varied simultaneously in a study of isocyanides and cyanides, including $\mathrm{HNC}$ and $\mathrm{HCN}$.

\section{CONCLUSIONS}

From SOFIA/EXES we present high spectral resolution, MIR absorption spectra of $\mathrm{HCN}, \mathrm{HNC}$, and $\mathrm{H}^{13} \mathrm{CN}$ towards the hot core Orion IRc2. This is the first MIR observations of $\mathrm{HNC}$ and $\mathrm{H}^{13} \mathrm{CN}$ in the ISM. Almost continuous coverage of the $\mathrm{P}, \mathrm{Q}$, and $\mathrm{R}$ branch transitions of $\mathrm{HNC}$, and the $\mathrm{Q}$ and $\mathrm{R}$ branch transitions of $\mathrm{HCN}$ yield detailed rotation diagrams that produce the species' excitation temperatures and column densities. Only three $\mathrm{H}^{13} \mathrm{CN} \mathrm{R}$ branch transitions are strong enough to include in a rotation diagram. All three species have a LSR velocity $-7 \mathrm{~km} \mathrm{~s}^{-1}$ with average temperatures and column densities for $\mathrm{HNC}$ of $98 \mathrm{~K}$ and $7.7 \times 10^{14} \mathrm{~cm}^{-2} ; \mathrm{H}^{13} \mathrm{CN} 97 \mathrm{~K}$ and $4.2 \times 10^{15} \mathrm{~cm}^{-2}$; and $\mathrm{HCN} 165 \mathrm{~K}$ and $5.5 \times 10^{16} \mathrm{~cm}^{-2}$. For the first time, we observe a second MIR velocity component of $\mathrm{HCN}$ measured at $1 \mathrm{~km} \mathrm{~s}^{-1}, 309 \mathrm{~K}$, and $1.6 \times 10^{16} \mathrm{~cm}^{-2}$.

Our $-7 \mathrm{~km} \mathrm{~s}^{-1}$ absorption lines belong to the same component measured in the MIR towards Orion IRc2 by Rangwala et al. (2018) and Lacy et al. (2005), which probes different material than the emission lines observed in the sub-mm/mm (Persson et al. 2007; Comito et al. 2005; Schilke et al. 2001, 1992; Stutzki et al. 1988; Blake et al. 1987). It is clear that the MIR is key to studying the ISM closest to the hot core compared to longer wavelength observations in sub- $\mathrm{mm} / \mathrm{mm}$. This $-7 \mathrm{~km} \mathrm{~s}^{-1}$ component is similar in velocity to a bipolar outflow originating from nearby radio source I, which has no infrared component. Our HCN, HNC, and $\mathrm{H}^{13} \mathrm{CN}$ are likely associated with this outflow.

The HCN in the $1 \mathrm{~km} \mathrm{~s}^{-1}$ component is much hotter and because of this, represents material closer to the hot core itself. EXES's small beam size compared to previous MIR and most grund-based sub- $\mathrm{mm} / \mathrm{mm}$ instruments allows us to isolate the material at the hot core's centre. While HNC only has one clear velocity component, its line widths are consistently wider than HCN, hinting that it may have two components, albeit unresolved. We would expect $\mathrm{HCN} / \mathrm{HNC}$ to be even higher in this hotter component. The $\mathrm{H}^{13} \mathrm{CN}$ lines do not have a high enough signal-to-noise to comment on the possibly of a second component.

We find $\mathrm{HCN} / \mathrm{HNC}=72 \pm 7$ in range of sub- $\mathrm{mm} / \mathrm{mm}$ observations towards Orion IRc2 (Schilke et al. 1992; Goldsmith et al. 1986). This supports findings that this ratio is enhanced in hot, irradiated environments. Our 
isotopic ratio ${ }^{12} \mathrm{C} /{ }^{13} \mathrm{C}=13 \pm 2$ is lower than expected for Orion IRc2's galactocentric distance, though similar to measurements by Rangwala et al. (2018) in the MIR and Tercero et al. (2010) in the $\mathrm{mm}$.

In order to determine the age of the hot core, we run the chemical network of Acharyya \& Herbst (2018) for a hot core model with three phases: free fall collapse, warmup, and post-warmup. Comparison with HCN and HNC abundances alone are highly uncertain given the range in observed $N_{\mathrm{H}_{2}}$ towards IRc2. Instead, we use our observed $\mathrm{HCN} / \mathrm{HNC}$ ratio to find that the hot core is $\sim 10^{6}$ years old. This supports similar results in Rangwala et al. (2018).

This work demonstrates the importance of the MIR in accessing transitions of $\mathrm{HCN}, \mathrm{HNC}$, and $\mathrm{H}^{13} \mathrm{CN}$ that originate in hotter material closer to the hot core compared to commonly used rotational transitions in the sub-mm/mm. The spectra presented here are a part of a larger survey with SOFIA/EXES towards Orion IRc2. Future papers detailing more molecular species are forthcoming. These studies will be critical in informing the observations from the James Webb Space Telescope (JWST), which while more sensitive, lacks the resolving power of SOFIA/EXES. With SOFIA/EXES we can unambiguously identify the signals from the strongest molecular species and construct a reference database to inform the search for weaker molecular species in JWST spectra.

\section{ACKNOWLEDGMENTS}

This work made use of the following Python packages: Astropy (Price-Whelan et al. 2018; Robitaille et al. 2013), Matplotlib (Hunter 2007), Numpy (van der Walt et al. 2011), Scipy (Virtanen et al. 2020), and HAPI (Kochanov et al. 2016). We would also like to thank James De Buizer for providing FORCAST data, and Kyle Kaplan for the useful conversation. SN gratefully acknowledges funding from the BAERI cooperative agreement 80NSSC17M0014. TJL, and XH gratefully acknowledge financial support from the NASA 16PDART16_2-0 080, 17-APRA17-0051, and 18-APRA180013 grants. $\mathrm{XH}$ acknowledges the NASA/SETI Cooperative Agreements NNX15AF45A, NNX17AL03G and 80NSSC19M0121. MND is supported by the Swiss National Science Foundation (SNSF) Ambizione grant 180079, the Center for Space and Habitability (CSH) Fellowship, and the IAU Gruber Foundation Fellowship. RCF acknowledges funding from NASA grant NNX17AH15G.

\section{REFERENCES}

Aalto, S., Garcia-Burillo, S., Muller, S., et al. 2012, A\&A, 537, 1, doi: 10.1051/0004-6361/201117919

Acharyya, K., \& Herbst, E. 2018, ApJ, 859, 51, doi: 10.3847/1538-4357/aabaf2

Aguado, A., Roncero, O., Zanchet, A., Agúndez, M., \& Cernicharo, J. 2017, ApJ, 838, 33, doi: 10.3847/1538-4357/aa63ee

Agúndez, M., Biver, N., Santos-Sanz, P., Bockelée-Morvan, D., \& Moreno, R. 2014, A\&A, 564, L2, doi: 10.1051/0004-6361/201423639

An, D., Ramírez, S. V., Sellgren, K., et al. 2009, ApJ, 702, 128, doi: 10.1088/0004-637X/702/2/L128

—. 2011, ApJ, 736, 133, doi: 10.1088/0004-637X/736/2/133

Bally, J., Ginsburg, A., Arce, H., et al. 2017, ApJ, 837, 60, doi: $10.3847 / 1538-4357 / \mathrm{aa} 5 \mathrm{c} 8 \mathrm{~b}$

Barber, R. J., Strange, J. K., Hill, C., et al. 2013, Monthly Notices of the Royal Astronomical Society, 437, 1828, doi: 10.1093/mnras/stt2011

Barr, A. G., Boogert, A., Dewitt, C. N., et al. 2018, ApJ: Letters, 868, L2, doi: 10.3847/2041-8213/aaeb23

Barr, A. G., Boogert, A., DeWitt, C. N., et al. 2020, arXiv e-prints. https://arxiv.org/abs/2007.11266
Belloche, A., Müller, H. S., Menten, K. M., Schilke, P., \& Comito, C. 2013, A\&A, 559, doi: 10.1051/0004-6361/201321096

Benson, P. J., \& Myers, P. C. 1989, ApJS, 71, 89

Bisschop, S. E., Jørgensen, J. K., van Dishoeck, E. F., \& De Wachter, E. B. 2007, A\&A, 465, 913, doi: 10.1051/0004-6361:20065963

Blake, G. A., Mundy, L. G., Carlstrom, J. E., et al. 1996, ApJ: Letters, 472, L49

Blake, G. A., Sutton, E. C., Masson, C. R., \& Phillips, T. G. 1987, ApJ, 315, 612

Bøgelund, E. G., Barr, A. G., Taquet, V., et al. 2019, A\&A, 628, A2, doi: 10.1051/0004-6361/201834527

Boonman, A. M., van Dishoeck, E. F., Lahuis, F., et al. 2003, A\&A, 399, 1047, doi: 10.1051/0004-6361:20021799

Boonman, A. M. S., Stark, R., van der Tak, F. F. S., et al. 2001, ApJ, 553, L63, doi: 10.1086/320493

Bottinelli, S., Ceccarelli, C., Lefloch, B., et al. 2004, ApJ, 615, 354, doi: 10.1086/423952

Bowman, J. M., Gazdy, B., Bentley, J. A., Lee, T. J., \& Dateo, C. E. 1993, The Journal of Chemical Physics, 99, 308, doi: 10.1063/1.465809 
Brown, P. D., Charnley, S. B., \& Millar, T. J. 1988, MNRAS, 231, 409

Bublitz, J., Kastner, J., Hily-Blant, P., et al. 2020, Galaxies, 8, 32, doi: 10.3390/galaxies 8020032

Bublitz, J., Kastner, J. H., Santander-García, M., et al. 2019, A\&A, 625, 1, doi: 10.1051/0004-6361/201834408

Bujarrabal, V., Fuente, A., \& Omont, A. 1994, A\&A, 285, 247

Cernicharo, J., Daniel, F., Castro-Carrizo, A., et al. 2013, ApJ: Letters, 778, L25, doi: 10.1088/2041-8205/778/2/L25

Cernicharo, J., Barlow, M. J., Gonzalez-Alfonso, E., et al. 1996, A\&AL, 204, L201

Cesaroni, R. 2005, in Proceedings of the International Astronomical Union, 59-69, doi: 10.1017/S1743921305004369

Chenel, A., Roncero, O., Aguado, A., Agúndez, M., \& Cernicharo, J. 2016, Journal of Chemical Physics, 144, doi: 10.1063/1.4945389

Clarke, M., Vacca, W. D., \& Shuping, R. Y. 2015, in Astronomical Data Analysis Software and Systems: XXIV, 355-358

Colzi, L., Fontani, F., Caselli, P., et al. 2018a, A\&A, 609, 1, doi: 10.1051/0004-6361/201730576

Colzi, L., Fontani, F., Rivilla, V. M., et al. 2018b, MNRAS, 478, 3693, doi: 10.1093/MNRAS/STY1027

Colzi, L., Sipilä, O., Roueff, E., Caselli, P., \& Fontani, F. 2020, A\&A. https://arxiv.org/abs/2006.03362

Comito, C., Schilke, P., Phillips, T. G., et al. 2005, ApJS, 156, 127, doi: 10.1086/425996

Crockett, N. R., Bergin, E. A., Neill, J. L., et al. 2014, ApJ, 787, 112, doi: 10.1088/0004-637X/787/2/112

Daniel, F., Gérin, M., Roueff, E., et al. 2013, A\&A, 560, 1, doi: 10.1051/0004-6361/201321939

De Buizer, J. M., Morris, M. R., Becklin, E. E., et al. 2012, ApJ: Letters, 749, 35, doi: 10.1088/2041-8205/749/2/L23

De Graauw, T., Haser, L. N., Beintema, D. A., et al. 1996, A\&A, 315, L47

Dungee, R., Boogert, A., Dewitt, C. N., et al. 2018, ApJ: Letters, 868, L10, doi: 10.3847/2041-8213/aaeda9

Dutrey, A., Guilloteau, S., \& Guélin, M. 1997, A\&A, 317, 55

Evans, N. J., Lacy, J. H., \& Carr, J. S. 1991, ApJ, 383, 674. file:///D:/TAABRARterbaru/TABATAFOAM/ jurnalpenelitian/BATUBATA.pdf

Favre, C., Despois, D., Brouillet, N., et al. 2011, A\&A, 532, 1

Favre, C., Carvajal, M., Field, D., et al. 2014, ApJS, 215, doi: 10.1088/0067-0049/215/2/25
Feng, S., Beuther, H., Henning, T., et al. 2015, A\&A, 590, C1, doi: 10.1051/0004-6361/201322725e

Gao, Y., \& Solomon, P. M. 2004, ApJS, 152, 63, doi: $10.1086 / 383003$

Genzel, R., Reid, M. J., Moran, J. M., \& Downes, D. 1981, ApJ, 244, 884, doi: 10.2307/2034794

Giesen, T. F., Mookerjea, B., Fuchs, G. W., et al. 2020, A\&A, 633, A120, doi: 10.1051/0004-6361/201936538

Goddi, C., Greenhill, L. J., Humphreys, E. M., Chandler, C. J., \& Matthews, L. D. 2011, ApJ: Letters, 739, L13, doi: 10.1088/2041-8205/739/1/L13

Goldsmith, P. F., Irvine, W. M., Hjalmarson, A., \& Ellder, J. 1986, ApJ, 310, 383, doi: $10.1017 / \mathrm{CBO} 9781107415324.004$

Goldsmith, P. F., \& Langer, W. D. 1999, ApJ, 517, 209, doi: $10.1086 / 307195$

Goldsmith, P. F., Langer, W. D., Ellder, Joel, Irvine, W., \& Kollberg, E. 1981, ApJ, 249, 524, doi: 10.1017/CBO9781107415324.004

Gómez, L., Rodríguez, L. F., Loinard, L., et al. 2008, ApJ, 685,333

Gong, Y., Henkel, C., Thorwirth, S., et al. 2015, A\&A, 581, 1, doi: 10.1051/0004-6361/201526275

Gordon, I. E., Rothman, L. S., Hill, C., et al. 2017, Journal of Quantitative Spectroscopy and Radiative Transfer, 203, 3, doi: 10.1016/j.jqsrt.2017.06.038

Graninger, D., Öberg, K. I., Qi, C., \& Kastner, J. 2015, ApJ: Letters, 807, L15, doi: 10.1088/2041-8205/807/1/L15

Graninger, D. M., Herbst, E., Öberg, K. I., \& Vasyunin, A. I. 2014, ApJ, 787, 74, doi: 10.1088/0004-637X/787/1/74

Guélin, M., Salomé, P., Neri, R., et al. 2007, A\&A, 462, 13, doi: 10.1051/0004-6361:20066555

Hacar, A., Bosman, A. D., \& van Dishoeck, E. F. 2020, A\&A, 635, A4, doi: 10.1051/0004-6361/201936516

Harada, N., Riquelme, D., Viti, S., et al. 2015, A\&A, 584, A102, doi: 10.1051/0004-6361/201526994

Harris, A. I., Avery, L. W., Schuster, K.-F., Tacconi, L. J., \& Genzel, R. 1995, ApJ, 446, L85, doi: 10.1086/187936

Harris, G. J., Pavlenko, Y. V., Jones, H. R., \& Tennyson, J. 2003, MNRAS, 344, 1107, doi: 10.1046/j.1365-8711.2003.06886.x

Harris, G. J., Tennyson, J., Kaminsky, B. M., Pavlenko, Y. V., \& Jones, H. R. 2006, Monthly Notices of the Royal Astronomical Society, 367, 400, doi: 10.1111/j.1365-2966.2005.09960.x

Henkel, C., Mauersberger, R., \& Schilke, P. 1988, A\&A, 201, L23 
Herbst, E. 1978, ApJ, 222, 508, doi: $10.1017 / \mathrm{CBO} 9781107415324.004$

Hernández Vera, M., Lique, F., Dumouchel, F., Hily-Blant, P., \& Faure, A. 2017, Monthly Notices of the Royal Astronomical Society, 468, 1084, doi: $10.1093 / \mathrm{mnras} / \mathrm{stx} 422$

Herpin, F., \& Cernicharo, J. 2000, ApJ, 530, L129, doi: $10.1086 / 312507$

Hirota, T., Kim, M. K., Kurono, Y., \& Honma, M. 2015, ApJ, 801, 82, doi: 10.1088/0004-637X/801/2/82

Hirota, T., Machida, M. N., Matsushita, Y., et al. 2017, Nature Astronomy, 1, 1, doi: 10.1038/s41550-017-0146

Hirota, T., Yamamoto, S., Mikami, H., \& Ohishi, M. 1998, ApJ, 503, 717

Ho, P. T. P., Barrett, A. H., Myers, P. C., et al. 1979, ApJ, 234, 912, doi: 10.1017/CBO9781107415324.004

Houck, J. R., Roellig, T. L., Van Cleve, J., et al. 2004, ApJ, 154, 18, doi: 10.1117/12.550517

Hrivnak, B. J., Volk, K., \& Kwok, S. 2000, ApJ, 535, 275, doi: $10.1086 / 308823$

Hunter, J. D. 2007, Computing in Science and Engineering, 9, 99, doi: 10.1109/MCSE.2007.55

Indriolo, N., Neufeld, D. A., DeWitt, C. N., et al. 2015a, ApJ, 802, L14, doi: 10.1088/2041-8205/802/2/L14

Indriolo, N., Neufeld, D. A., Gerin, M., et al. 2015b, ApJ, 800, 40, doi: 10.1088/0004-637X/800/1/40

Indriolo, N., Neufeld, D. A., Barr, A. G., et al. 2020, ApJ, 894, 107, doi: 10.3847/1538-4357/ab88a1

Irvine, W. M., \& Schloerb, F. P. 1984, ApJ, 282, 516

Jacquinet-Husson, N., Armante, R., Scott, N. A., et al. 2016, Journal of Molecular Spectroscopy, 327, 31, doi: 10.1016/j.jms.2016.06.007

Jin, M., Lee, J. E., \& Kim, K. T. 2015, ApJ, 219, 2, doi: 10.1088/0067-0049/219/1/2

Jørgensen, J. K., Müller, H. S., Calcutt, H., et al. 2018, A\&A, 620, A170, doi: 10.1051/0004-6361/201731667

Kastner, J. H., Zuckerman, B., Weintraub, D. A., \& Forveille, T. 1997, Science, 277, 67, doi: 10.1126/science.277.5322.67

Knez, C., Boonman, A. M. S., Lacy, J. H., Evans, N. J., I., \& Richter, M. J. 2001, in American Astronomical Society Meeting Abstracts, Vol. 199, American Astronomical Society Meeting Abstracts, 134.10

Knez, C., Lacy, J. H., Evans, N. J., Van Dishoeck, E. F., \& Richter, M. J. 2009, ApJ, 696, 471, doi: 10.1088/0004-637X/696/1/471

Kochanov, R. V., Gordon, I. E., Rothman, L. S., et al. 2016, Journal of Quantitative Spectroscopy and Radiative Transfer, 177, 15, doi: 10.1016/j.jqsrt.2016.03.005
Kounkel, M., Hartmann, L., Loinard, L., et al. 2017, APJ, 834, 142, doi: 10.3847/1538-4357/834/2/142

Lacy, J. H., Knez, C., Evans, N. J., \& Richter, M. J. 2005, in American Astronomical Society Meeting Abstracts, Vol. 207, American Astronomical Society Meeting Abstracts, 81.22

Lacy, J. H., Richter, M. J., Greathouse, T. K., Jaffe, D. T., \& Zhu, Q. 2002, PASP, 114, 153, doi: 10.1086/338730

Lahuis, F., \& van Dishoeck, E. F. 2000, A\&A, 355, 699

Lahuis, F., van Dishoeck, E. F., Boogert, A. C. A., et al. 2006, ApJ, 636, L145, doi: 10.1086/500084

Lee, T. J., \& Rendell, A. P. 1991, Chemical Physics Letters, 177, 491, doi: 10.1016/0009-2614(91)90073-I

Lis, D. C., Keene, J., Young, K., et al. 1997, Icarus, 130, 355, doi: 10.1006/icar.1997.5833

Liszt, H., \& Lucas, R. 2001, A\&A, 370, 576

Lo, J. I., Chou, S. L., Peng, Y. C., et al. 2015, ApJS, 221, 20, doi: 10.1088/0067-0049/221/1/20

Loison, J. C., Wakelam, V., \& Hickson, K. M. 2014, MNRAS, 443, 398, doi: 10.1093/mnras/stu1089

Lord, S. D. 1992, A New Software Tool for Computing Earth's Atmospheric Transmission of Near- and Far-Infrared Radiation. https://atran.arc.nasa.gov/cgi-bin/atran/atran.cgi

Loughnane, R. M., Redman, M. P., Thompson, M. A., et al. 2012, MNRAS, 420, 1367, doi: 10.1111/j.1365-2966.2011.20121.x

Magalhães, V. S., Hily-Blant, P., Faure, A., Hernandez-Vera, M., \& Lique, F. 2018, A\&A, 615, 1, doi: 10.1051/0004-6361/201832622

McGuire, B. A. 2018, ApJS, 239, 17, doi: 10.3847/1538-4365/aae5d2

Mendes, M. B., Buhr, H., Berg, M. H., et al. 2012, ApJ: Letters, 746, 3, doi: 10.1088/2041-8205/746/1/L8

Miettinen, O. 2014, A\&A, 562, 1, doi: 10.1051/0004-6361/201322596

Milam, S. N., Savage, C., Brewster, M. A., Ziurys, L. M., \& Wyckoff, S. 2005, ApJ, 634, 1126, doi: 10.1086/497123

Moreno, R., Lellouch, E., Lara, L. M., et al. 2011, A\&AL, 536, L12, doi: 10.1051/0004-6361/201118189

Nguyen, T. L., Baraban, J. H., Ruscic, B., \& Stanton, J. F. 2015, Journal of Physical Chemistry A, 119, 10929, doi: 10.1021/acs.jpca.5b08406

Ohishi, M. 1997, in International Astronomical Union Symposium, 61-74

Okumura, S. I., Yamashita, T., Sako, S., et al. 2011, Publ. Astron. Soc. Japan, 63, 823, doi: 10.1093/pasj/63.4.823

Orozco-Aguilera, M. T., Zapata, L. A., Hirota, T., Qin, S.-L., \& Masqué, J. M. 2017, ApJ, 847, 66, doi: $10.3847 / 1538-4357 / \mathrm{aa} 88 \mathrm{~cd}$ 
Peng, Y., Rivilla, V. M., Zhang, L., Ge, J. X., \& Zhou, B. 2019, ApJ, 871, 251, doi: 10.3847/1538-4357/aafad4

Pérez-Beaupuits, J. P., Aalto, S., \& Gerebro, H. 2007, A\&A, 476, 177, doi: 10.1051/0004-6361:20078479

Persson, C. M., Olofsson, A. O. H., Koning, N., et al. 2007, A\&A, 476, 807, doi: 10.1051/0004-6361:20077229

Plambeck, R. L., \& Wright, M. C. H. 2016, ApJ, 833, 219, doi: $10.3847 / 1538-4357 / 833 / 2 / 219$

Plambeck, R. L., Wright, M. C., Friedel, D. N., et al. 2009, ApJ, 704, 28, doi: 10.1088/0004-637X/704/1/L25

Plume, R., Bergin, E. A., Phillips, T. G., et al. 2012, ApJ, 744, doi: 10.1088/0004-637X/744/1/28

Price-Whelan, A. M., Sipcz, B. M., Günther, H. M., et al. 2018, ApJ, 156, 123, doi: 10.3847/1538-3881/aabc4f

Rangwala, N., Colgan, S. W. J., Le Gal, R., et al. 2018, ApJ, 856, 9, doi: 10.3847/1538-4357/aaab66

Richter, M. J., DeWitt, C. N., McKelvey, M., et al. 2018, Journal of Astronomical Instrumentation, 7, 1, doi: 10.1142/S2251171718400135

Rickard, L. J., Palmer, P., Turner, B. E., Morris, M., \& Zuckerman, B. 1977, ApJ, 214, 390

Rivilla, V. M., Beltrán, M. T., Cesaroni, R., et al. 2017, A\&A, 598, 1, doi: 10.1051/0004-6361/201628373

Robitaille, T. P., Tollerud, E. J., Greenfield, P., et al. 2013, A\&A, 558, 1, doi: 10.1051/0004-6361/201322068

Rolffs, R., Schilke, P., Wyrowski, F., et al. 2011, A\&A, 529, 1, doi: 10.1051/0004-6361/201116544

Sarrasin, E., Ben Abdallah, D., Wernli, M., et al. 2010, Monthly Notices of the Royal Astronomical Society, 404, 518, doi: 10.1111/j.1365-2966.2010.16312.x

Schilke, P., Benford, D. J., Hunter, T. R., Lis, D. C., \& Phillips, T. G. 2001, ApJS, 132, 281, doi: 10.1086/318951

Schilke, P., Groesbeck, T. D., Blake, G. A., \& Phillips, T. G. 1997, ApJS, 108, 301, doi: 10.1086/312948

Schilke, P., Walmsley, C. M., G. Pineau des Forêts, et al. 1992, A\&A, 256, 595

Shuping, R. Y., Morris, M., \& Bally, J. 2004, AJ, 128, 363, doi: 10.1086/421373

Snyder, L. E., \& Buhl, D. 1971, ANYAS, 194, 17

—. 1972, ApJ, 177, 619, doi: 10.1086/151739

Stutzki, J., Genzel, R., Harris, A. I., Herman, J., \& Jaffe, D. T. 1988, ApJ, 330, L125, doi: 10.1086/185219

Sutton, E. C., Peng, R., Danchi, W. C., et al. 1995, ApJS, 97,455
Tennekes, P. P., Harju, J., Juvela, M., \& Tóth, L. V. 2006, A\&A, 456, 1037, doi: 10.1051/0004-6361:20040294

Tercero, B., Cernicharo, J., Pardo, J. R., \& Goicoechea, J. R. 2010, A\&A, 517, doi: 10.1051/0004-6361/200913501

Turner, B. E., Pirgov, L., \& Minh, Y. C. 1997, ApJ, 483, 235

van der Tak, F. F. 2004, in Star Formation at High Angular Resolution, IAU Symposium, 59-66

van der Walt, S., Colbert, S. C., \& Varoquaux, G. 2011, Computing in Science and Engineering, 13, 22, doi: 10.1109/MCSE.2011.37

van Dishoeck, E. F., Wright, C. M., Helmich, F. P., et al. 1998, ApJ, 502, 1996

Vasyunina, T., Linz, H., Henning, T., et al. 2011, A\&A, 527, A88, doi: 10.1051/0004-6361/201014974

Virtanen, P., Gommers, R., Oliphant, T. E., et al. 2020, Nature Methods, 17, 261, doi: 10.1038/s41592-019-0686-2

Wampfler, S. F., Jørgensen, J. K., Bizzarro, M., \& Bisschop, S. E. 2014, A\&A, 572, 1, doi: 10.1051/0004-6361/201423773

Willis, E. R., Garrod, R. T., Belloche, A., et al. 2020, A\&A, 636, 1, doi: 10.1051/0004-6361/201936489

Wright, M. C. H., \& Plambeck, R. L. 2017, ApJ, 843, 83, doi: 10.3847/1538-4357/aa72e6

Wright, M. C. H., Plambeck, R. L., Mundy, L. G., \& Looney, L. W. 1995, ApJ, 455, doi: 10.1086/309829

Yan, Y. T., Zhang, J. S., Henkel, C., et al. 2019, ApJ, 877, 154, doi: 10.3847/1538-4357/ab17d6

Young, E. T., Becklin, E. E., Marcum, P. M., et al. 2012, ApJ: Letters, 749, 5, doi: 10.1088/2041-8205/749/2/L17

Zapata, L. A., Rodríguez, L. F., Schmid-Burgk, J., et al. 2012, ApJ, 754, doi: 10.1088/2041-8205/754/1/L17

Zapata, L. A., Schmid-Burgk, J., \& Menten, K. M. 2011, Astronomy and Astrophysics, 529, 1, doi: 10.1051/0004-6361/201014423

Zeng, S., Jiménez-Serra, I., Cosentino, G., et al. 2017, A\&A, 603, 1, doi: 10.1051/0004-6361/201630210

Zhang, X., Quan, D., Chang, Q., et al. 2020, MNRAS, 497, 609, doi: 10.1093/mnras/staa1979

Zuckerman, B., Morris, M., Palmer, P., \& Turner, B. E. 1972, APJ, 173, L125 